
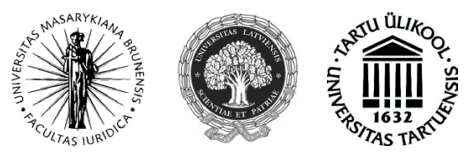

ISSN 1392-6195 (print) ISSN 2029-2058 (online) JURISPRUDENCIJA JURISPRUDENCE 2013, 20(3), p. 971-1003.

\title{
AMBIVALENCES EN DROIT DE LA CONSOMMATION REMARQUES SUR LES EFFETS DE L'ÉCRITURE DU DROIT
}

\author{
Vincent Forray* \\ Université McGill - Faculté de droit \\ 3644, rue Peel \\ Montréal, Québec, Canada H3A 1W9 \\ vincent.forray@mcgill.ca
}

Reçu le 28 juin 2013. Prêt pour la presse le 25 septembre 2013.

doi:10.13165/JUR-13-20-3-07

Partout où il se trouve, le droit de la consommation signale une série de problèmes qu'il s'emploie à régler : des déséquilibres, des injustices, des iniquités. Il signifie donc au droit qui l'entoure l'incapacité préalable de celui-ci à résoudre de telles questions. Le droit de la consommation, du seul fait de son existence, juge le système qui lui préexiste.

Si on le considère, non plus isolément, mais comme une partie du système, une opposition se dessine. Il y a, d'une part, le discours fondateur du droit de la consommation qui le représente comme un droit à part, un droit formé à la justice sociale ou à la régulation du marché ; un droit volontiers subversif de l'ordre juridique qui le précède. Il y a, d'autre part, l'ajustement du droit de la consommation aux catégories juridiques générales, son intégration au droit commun, son inscription dans le cour du droit privé dont il paraissait annoncer le bouleversement. Une distance s'établit entre l'intention initiale qui soutient le corpus

Ce texte a été écrit à la suite d'une conférence organisée par l'Association Québécoise de Droit Comparé et prononcée à l'Université McGill le 18 mai 2012. Je remercie Nathalie Vézina et Daniel Boyer pour leur invitation, ainsi que les participants pour leurs remarques et questions. Je veux aussi remercier Michelle Cumyn, H. Patrick Glenn, Daniel Jutras, Robert Leckey, Sébastien Pimont et Ruth Sefton-Green pour leurs précieux commentaires et suggestions sur ce texte. Toutes les erreurs sont les miennes. 
consumériste et les effets produits par celui-ci. Je soutiens dans cet article que cette distance, qui se creuse jusqu'à introduire l'ambivalence au sein du droit en question, est un effet propre du mode de formalisation juridique privilégié dans les sociétés occidentales contemporaines: l'écriture.

Mots-clés : droit de la consommation - écriture - consommateur - citoyen - critique ordre juridique - formalisation.

Les subalternes peuvent-ils parler? Que doit faire l'élite pour prévenir la construction continue des subalternes?

Protocole

Gayatri Chakravorty Spivak

L'irruption - L'écriture

Un ensemble normatif nouveau ne semble pas pouvoir faire irruption dans les ordres juridiques sans les compromettre en tant qu'ordres juridiques. Une nouveauté du droit renvoie à une série de problèmes qu'elle s'emploie à régler : des déséquilibres, des injustices, des iniquités, un désordre. Elle signifie au droit qui l'entoure l'incapacité préalable de celui-ci à résoudre de telles questions. Il paraît difficile de dire le droit nouveau sans prendre parti sur le droit ancien, sans juger le système qui préexiste. Et pourtant, le droit civil est toujours là ; le common law est toujours là. Ils doivent avoir quelque chose d'immuable qui fait qu'ils demeurent le droit civil et le common law. Une forme d'immuabilité qualifie les systèmes de droit, peut-être plus que tout le reste.

Pourtant les droits en question changent ; ils évoluent. Ils ont une histoire que l'on peut raconter. Le récit existe de ce qui n'est plus en droit civil ou en common law (la prison pour dettes, l'esclavage, le supplice), de leurs modifications (les Constitutions, les lois, les modes de preuve), de leurs nouveautés (les législations sur les clauses abusives, le droit d'auteur dans le domaine numérique). Les institutions, les concepts, les règles, les pratiques, les pouvoirs, les sources, les autorités ; tout ce qui correspond pendant un temps à ce qu'on appelle le droit disparaît ou se transforme. Le droit est en mouvement.

Et cependant, il semble disposer d'une aptitude remarquable à recomposer l'allure qu'on lui connaît. En dépit des outrages du temps ou de l'injustice. Comme si le droit produisait en son sein et par lui-même ce qui lui permet de changer sans changer ${ }^{1}$. D'être lui et tout ce qu'il a été .

1 Karl. N. Llewellyn, The Theory of rules, Edited and with an Introduction of Frederick Schauer, The University of Chicago Press, 2011, aux p. 139-151, spéc. : « For stability in things of law is not the absence of change. Stability consists rather of sufficient order in change, sufficient primitive approach to imperceptibility in change, to make change non-disturbing, even anticipated as it occurs - to make any failure of the impending change be felt as a disturbance, as a pinching by the past, as a « technicality » in that invidious sense which means that it comes to notice only as thing which ought already to be ironed out ».

2 H. Patrick Glenn, Legal Traditions of the World, 4th ed., Oxford University Press, 2010, p. 1-27. Pour une justification de type phénoménologique à l'immuabilité du droit (par opposition à l'idée de son «perpétuel 
Les expressions « droit civil » et «common law » ont certainement une autre fonction que de signaler des systèmes temporalisés - le droit en vigueur, et localisés tel ou tel ensemble. Elles indiquent aussi ce que chacun des « systèmes », chacune des « traditions », chacune des « cultures » juridiques ont d'immuable.

L'immuabilité a son siège dans un mouvement interminable de formalisation. En effet, l'ordre ou le système juridique, l'institution et la connaissance du droit se signalent par des formes. Il faut fixer le droit pour que celui-ci « dure au-delà de l'instant ». En fixer les formules, les codes, les règles et les évènements pour qu'il soit appréhendable. En fixer les commentaires et les interprétations pour qu'il soit applicable. En fixer les principes et les concepts pour qu'il soit compréhensible. Il faut formaliser le droit pour qu'il soit, tout simplement.

La formalisation consiste dans le mouvement le plus banal de mise en forme du droit dans une société. Le geste qui pose le droit comme intelligible. Elle désigne aussi un mouvement permanent. Car les formes juridiques prolifèrent, comme si la mise en forme du droit n'était jamais achevée. Il y a toujours d'autres règles, d'autres interprétations, d'autres commentaires. Le droit n'en finit pas d'être mis en forme.

Selon les traditions juridiques, les instances de formalisation du droit varient. En revanche, si l'on s'en tient aux systèmes de droit civil et de common law, il est possible de soutenir que le medium de la formalisation du droit est toujours le même : l'écriture. Et à condition de considérer l'écriture dans son sens le plus étendu, selon la conception développée par Jacques Derrida. Pour couper au plus court, selon le philosophe, il n'y a pas d'écriture au seul cas de production d'un texte, mais à chaque fois qu'il y a trace ${ }^{3}$. Tout ce qui est communiqué en dehors de la présence du locuteur participe de l'écriture. Très peu de dispositifs du droit échappent alors à celle-ci : ceux qui ne laissent pas de trace et dont la consistance juridique demeure, par conséquent, une question. Que vaut, en droit, le droit dont on a perdu la mémoire ? A l'inverse les discours oraux, lorsqu'ils sont conservés sous une forme ou sous une autre, et même ceux écrits pour être dits, constituent de l'écriture. La plaidoirie ou les mots prononcés par un professeur de droit dans un cours concourent à l'écriture de l'argument de droit, de la décision ou de la connaissance juridique. Il faut aussi comprendre dans l'écriture les signes, les chiffres et les symboles du droit.

Pourquoi retenir une conception aussi large de l'écriture ? Parce qu'elle permet de regrouper les formes juridiques sous l'angle de ce qu'elles ont en commun : d'avoir été formalisées. Or, et c'est l'hypothèse développée dans le présent texte, si la formalisation nous dit quelque chose du droit, quelque chose de fondamental et de préalable à tout système de classement, et que cette formalisation passe par l'écriture, nous devrions pouvoir profiter des connaissances accumulées par ailleurs sur l'écriture afin d'essayer de comprendre ce que celle-ci fait au droit.

mouvement ou d'une perpétuelle évolution ») voir Adolf Reinach, Les fondements a priori du droit civil, traduit de l'allemand par Ronan de Calan, Paris, Vrin, 2004.

3 De la grammatologie, Paris, Les Editions de Minuit, 1967 ; La voix et le phénomène, Introduction au problème du signe dans la phénoménologie de Husserl, Paris, 1967, 4 ${ }^{\text {ème }}$ éd., P.U.F., 2009. 
Relions maintenant le sentiment de ce que l'ordre ou le système juridique a quelque chose d'immuable avec le phénomène de formalisation du droit. On en vient à penser que c'est celui-ci qui fait se tenir le droit tel qu'il est. Que l'écriture, donc, est pour beaucoup dans la constitution de l'ordre ou du système. Qu'elle garantit au droit sa quiétude. Elle inscrit, elle construit, elle resserre, elle contient. Elle présente les idées, les concepts et les règles juridiques dans l'ordre de la forme. Les expressions « droit civil » et « common law » désignent, aussi, un ordre de l'écriture du droit, un ensemble stabilisé par l'écriture.

Il aura bien fallu, alors, réduire les écarts, dénouer les tensions, lutter pour l'ordre. Parce que la paix du texte est provisoire, fugace même. Toujours, le questionnement surgit, et la menace. La question de droit compromet l'immuable juridique, précisément parce qu'elle le désigne :

« Dans le rapport entre question de droit et réponse juridique s'insinue l'aporie irrésolue depuis toujours. La réponse est juridique parce qu'elle est trouvée dans un droit établi, « positif ». Ce droit positif n'est pourtant pas contenu dans l'ensemble des règles consacrées. Il se découvre jour après jour, espèce après espèce, moyennant la mise en œuvre de principes relativement indéterminés et de méthodes toujours relativement incertaines. Il « juge » les réponses qui le « jugent» en lui donnant sens, en lui donnant son seul sens $»^{4}$.

La question de droit est donc celle qui anime le droit en indiquant une " aporie », c'est-à-dire une contradiction dont les termes sont également raisonnés. De sorte que la question ouvre le droit ; elle le scinde. Elle enveloppe, forcément, un reproche adressé au droit — sans quoi il n'y aurait pas de question—, mais elle le fait en droit. Il faut que la question ait forme de droit. Elle doit user du vocabulaire juridique et de la grammaire du droit ${ }^{5}$. Il lui faut s'approprier la forme du droit; elle s'écrit, elle aussi, en droit.

A l'inverse, le droit n'est pas inquiété par des questions qui ne disposent pas de la forme juridique. Ou des questions que ne se posent pas des juristes ${ }^{6}$. De telles questions, qui peuvent être graves, ne touchent le droit qu'à sa périphérie. Elles se forment à l'extérieur de son domaine de propriété et rôdent à ses frontières. Nous les apercevons. Nous savons que les questions de justice, de pouvoir, d'éthique, de morale, d'économie concernent (ont un rapport avec) le droit ; peut-être même qu'elles encerclent et contiennent celui-ci. Mais nous savons aussi qu'il leur demeure irréductible. Nous, juristes, ne traitons pas le droit comme s'il était uniquement la forme normative, discursive ou stipulative de telles questions. Poser ces questions en droit suppose leur migration préalable. Il s'agit d'engager un processus qui n'est pas une simple transposition. Plutôt une transformation ou une conversion. Il faut que la question chemine jusqu'à la forme juridique. Qu'elle trouve dans le droit un véhicule

4 Christian Atias, Questions et réponses en droit, Paris, P.U.F., 2009, p. 70-71.

5 Voir Desmond Menderson, Proximity, Levinas, and the Soul of Law, McGill-Queen's University Press, 2006, p. 21-25.

6 « Economists are very good indeed at framing questions that lawyers and consumer advocates have not asked », M. Pertschuk (former Chair of the United States Federal Trade Commission), cité par Ian Ramsay, Consumer Law and Policy, Text and Materials on Regulating Consumer Markets, $2^{\text {nd }}$ ed., Oxford and Portland, Hart Publishing, 2007, p. 55. 
formel : le procès (la question chemine par les procédures judiciaires), une théorie (la question prend forme selon les modes de rationalisation familiers aux juristes ${ }^{7}$, les rites disciplinaires et communautaires d'écriture), la loi (la question progresse dans les procédures parlementaires). Dans tous les cas, la question originaire se $\langle\text { perd }\rangle^{8}$. Elle est enfouie dans la forme juridique. Ou bien elle s'est scindée entre la question restée hors du (texte du) droit et celle venue dans le droit. Cette fragmentation des questions est familière aux juristes. Eux sont tenus d'obéir depuis longtemps aux assignations d'une pensée plus ample que leurs discours ${ }^{9}$. « Quid juris ?» ${ }^{10}$.

La constitution d'un énoncé de droit implique l'intervention de l'écriture, capable de fixer ce qui est dit et de le faire conformément au vocabulaire ainsi qu'à la grammaire juridiques. «Il n'y a pas de droit sans possibilité de trace ${ }^{11}$. L'écriture démarque le droit de l'ensemble des savoirs et des pratiques mobilisés par les questions qui ne sont pas de droit. Elle opère aussi une soustraction de l'interrogation dérangeante pour le droit. D'où le fait que le droit ne se présente pas comme un ensemble d'interrogations mais d'affirmations, des « réponses sans questions » comme le dit Christian Atias. La représentation du droit positif, c'est-à-dire formellement stabilisé, est à ce prix.

Il faut alors reconsidérer l'irruption. En remarquant qu'il n'est pas possible de la caractériser par la brutalité de l'apparition d'une forme juridique. Celle-ci n'émerge qu'au terme d'un encodage qui a pour conséquence d'en neutraliser l'urgence, d'en dissimuler les dangers, d'en conjurer la subversion. Une violence, celle de l'irruption de la nouveauté juridique, est contrebalancée par une autre violence porteuse de légitimité, celle de l'écriture du droit.

L'entrée de nouvelles règles ou d'interprétations dans l'ordre juridique semble donc naturelle. Comme si l'évolution était préparée de longue date par la totalité du droit. C'est le cas d'un certain point de vue. L'élaboration constante des catégories, l'établissement permanent des hiérarchies et l'aménagement continu des espaces font que la nouveauté n'est pas une surprise ${ }^{12}$. Tout est fait pour que rien ne surprenne le droit.

7 Max Weber, Sociologie du droit, préface Philippe Raynaud, introduction et traduction par Jacques Grosclaude, Paris, P.U.F., 2007, spéc. p. 162-168 et 219-235 ; voir aussi Duncan Kennedy, The Disenchantment of Logically Formal Legal Rationality, or Max Weber's Sociology in the Genealogy of the Contemporary Mode of Western Legal Thought, (2004) 55 Hastings L. J. 1031.

Sur un autre plan, Michelle Cumyn, Les catégories, la classification et la qualification juridiques : réflexions sur la systématicité du droit, (2011) 52 Les Cahiers de Droit 351, spéc. aux p. 368-378.

8 Christian Atias, supra note 4.

9 Emmanuel Kant, Le conflit des Facultés en trois sections, traduction, introduction et notes par J. Gibelin,

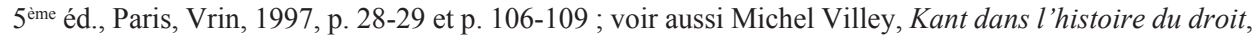
in Leçons d'histoire de la philosophie du droit, réédition présentée par René Sève, Paris, Dalloz, 2002, p. 251-269, spéc. p. 251-261.

10 Christian Atias, supra note 4 aux p. 21-23 ; voir l'intéressant renouvellement (kantien) de la question proposée par Simone Goyard-Fabre, Re-penser la pensée du droit, Les doctrines occidentales modernes au tribunal de la raison interrogative critique, Paris, Vrin, 2007, p. 135-146.

11 Jacques Derrida, De la grammatologie, supra note 3, p. 141.

12 Voir Michelle Cumyn, « La classification des catégories juridiques en droit comparé. Métaphores taxonomiques », (2008) R. du N. 1 (erratum) (numéro spécial du Xe Congrès de l'Association internationale de méthodologie juridique sur la sécurité juridique). 
Il n'empêche que le fait de l'irruption demeure. Quoique l'écriture joue pleinement, il reste au texte une marque silencieuse des reproches encourus par le droit. L'irruption est subversive dans son moment propre.

Et ainsi, en chaque forme juridique se trouve l'instruction d'un procès du droit. En s'écrivant, le droit conserve la mémoire de ses ambivalences. L'écrit est bien une cicatrice et l'écriture, une cautérisation. Un tissu du droit est brièvement abîmé pour préserver le corpus juris. Un texte juridique signale le reproche fait au droit en même temps qu'il soustrait le droit de l'ensemble des conséquences que l'on pourrait vouloir tirer d'un tel reproche. Une question de droit, lorsqu'elle formule l'interrogation juridique qui doit, à son terme, éconduire le questionnement originaire, met le droit en accusation. Elle participe à l'institution du droit ambivalent.

\section{Introduction}

La formalisation du «droit de la consommation » a eu lieu dans la plupart des systèmes de droit civil et de common law $w^{13}$. Du point de vue des traditions juridiques en question, l'émergence du droit de la consommation a quelque chose de l'irruption, telle que les propos précédents l'ont esquissée. Le droit de la consommation s'élève sur un reproche fait au droit préexistant. Il signifie au moins à ce dernier son inaptitude à résoudre les problèmes posés par la "société de consommation », comme l'ont rappelé les juges LeBel et Cromwelldans une décision de la Cour Suprême du Canada : "L'avènement de la société de consommation a rendu évidentes les limites du droit commun au Québec comme dans les autres provinces canadiennes $\rangle^{14}$. Le droit de la consommation pourrait aussi indiquer l'échec d'une communauté juridique toute entière ${ }^{15}$.

En outre, pareil à celui qui fait irruption, le droit de la consommation se présente comme un étranger dans les droits qui l'accueillent. Il est un Autre. Un Autre porteur de l'image d'un monde en train d'advenir ${ }^{16}$. Porteur aussi d'une promesse : l'économie de marché totale et apaisée, jumelée - enfin - aux exigences de la démocratie ; l'achèvement d'un projet libéral moderne ${ }^{17}$.

13 Pour avoir une idée de l'amplitude du phénomène, voir par ex. Ian Ramsay (éditeur), Consumer Law in the Global Economy, Ashgate, Dartmouth, 1997 ; William T. Vukowich, Consumer Protection in the 21st Century, A global Perspective, Transnational Publishers, 2002.

14 Richard c. Time Inc., 2012 CSC 8, par. 39.

15 «L'incapacité de la doctrine et de la jurisprudence à formuler une réponse adéquate aux problèmes créés par le contrat d'adhésion serait, selon Claude Masse, à l'origine même du droit québécois de la consommation. L'échec théorique et pratique de la communauté juridique rendait indispensable l'intervention législative de l'Etat », Jean-Guy Belley, « La Loi sur la protection du consommateur comme archétype d'une conception socioéconomique du contrat ", dans Pierre-Claude Lafond, dir., Mélanges Claude Masse, En quête de justice et d'équité, Cowansville, Editions Yvon Blais, 2003, à la p. 124.

16 Allan C. Hutchinson, "Life After Shopping : From Consumers to Citizens ", dans Ian Ramsay, dir., Consumer Law in the Global Economy, National and International Dimensions, Ashgate Dartmouth, 1997, p. 25.

17 Voir la place faite au discours de John F. Kennedy dans les introductions au droit de la consommation,

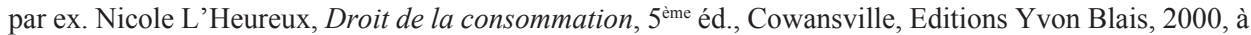

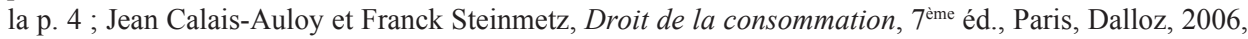


Le droit de la consommation, présent en droit civil et en common law, se démarque de l'une et l'autre des traditions au sein desquelles il apparaît singulier. De ce fait, il exige la comparaison ; la comparaison comme entreprise discursive ou dialogique. Sans développer ici un propos comparatiste, je solliciterai la comparaison comme approche de l'altérité, comme ce qui nous offre une chance de devenir « étrangers à nous-mêmes " ${ }^{18}$. J'emprunterai des matériaux à différentes littératures juridiques : Canada et Québec, France, Angleterre, Etats-Unis et Union Européenne.

Le discours initial du droit de la consommation est un discours de la différence ${ }^{19}$. Le droit de la consommation n'est pas le droit dominant ou le droit commun. Il présente des dispositions critiques à leur égard. Il entre dans son principe d'en corriger les défauts qui apparaissent à l'occasion de certains rapports économiques. Le droit de la consommation est « spécial ». Le type civiliste du « droit spécial » ne doit pas tromper. Le discours initial du droit de la consommation se prépare aussi en common law ${ }^{20}$.

Quelle que soit la tradition juridique, un tel discours inaugure une idéologie du droit de la consommation. J'entends par là un propos préparatoire, indiquant la situation du droit dont on s'apprête à exposer les techniques ${ }^{21}$. A cet égard, remarquons quel est le lieu privilégié de ce discours : les préfaces ou les introductions des livres de droit de la consommation (ce qui est « hors-livre » ${ }^{22}$ ?).

Je soutiendrai dans ce qui suit que ce discours initial, dépositaire des intentions subversives du droit de la consommation, n'est finalement que protocolaire. Indexant sa charge critique sur le droit dont il se distingue, et en exposant son propre potentiel subversif, il fournit un principe de stabilisation. Il offre des possibilités de transaction. Le droit dominant ne peut pas être aussi déséquilibré, injuste ou inéquitable que le discours initial du droit de la consommation dit qu'il l'est. Parce que le droit de la consommation prend forme dans les matériaux du droit dont il s'était préalablement démarqué ou, tout au moins, décentré. Il s'écrit avec le droit dominant.

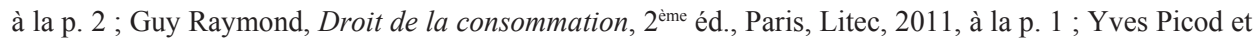

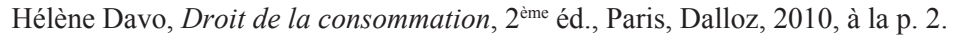

18 Julia Kristeva, Etrangers à nous-mêmes, Paris, Gallimard, 1988, aux p. 269-285.

19 Thierry Bourgoignie, «Un droit de la consommation est-il encore nécessaire en 2006 », dans Thierry Bourgoignie, dir., Regards croisés sur les enjeux contemporains du droit, Cowansville, Editions Yvon Blais, 2006, p. 1.

20 On en trouve les linéaments dans le casebook fondateur de John A. Spanogle et al., Consumer Law, Cases and Materials, 3ème ed., Thomson West, 2007, voir la préface à la première édition et l'introduction, spéc. à la p. 1 ; voir aussi Geraint Howells et Stephen Weatherhill: « Private law has been shaped and bolstered by the judges and by the (UK and EC) legislature to adjust the pattern of market relations, and, sometimes directly, sometimes indirectly, to protect the consumer. This has done much to create a discernible framework of consumer protection law within the private law. However, it will be shown that some markets require public regulation, for reasons associated with the inadequacies of private law to achieve a free and fair market », Consumer Protection Law, Ashgate 2005, à la p. 9.

21 Un peu à la manière dont Edmund Husserl veut «préparer l'accès » à la phénoménologie pure « en caractérisant sa situation exceptionnelle » dans les Idées directrices pour une phénoménologie, trad. Paul Ricoeur, Paris Gallimard, 1950, réimpr. 2008, à la p. 3. 
De sorte que le droit de la consommation vient, à son tour, constituer le droit dominant. Au-delà de son discours protocolaire, il est ambivalent. Droit de la consommation et droit dominant ou droit commun présentent un front uni au sein duquel chacun respecte les différences de l'Autre (Un Autre qui n'est que l'image de Soi renversée). Si on accepte la possibilité de ce cheminement, on verra que le discours annonçant la subversion du droit de la consommation s'épuise au terme de son annonce.

Ce n'est pas que le discours du droit de la consommation manque son but. Mais il s'absorbe dans une certaine intentionnalité, un "vouloir-dire $»^{23}$, qui ne prend pas en considération l'écriture juridique, cette « codification continue $»^{24} \mathrm{du}$ droit. Celle-ci implique les mots et la grammaire du droit dominant ; elle mobilise les catégories, les qualifications et les hiérarchies qui fixent le discours dans sa forme juridique. Le droit de la consommation ne peut se développer sans le soutien d'une composition formelle qui le sature du droit dont il avait pourtant vocation à s'écarter.

Il y donc deux mouvements simultanés du droit de la consommation qui l'installent dans l'ambivalence : un mouvement subversif opéré par le discours qui l'annonce et un mouvement de l'écriture du droit qui vient conjurer cette subversion. Cet article tente d'ouvrir une perspective sur ces deux mouvements. Dans une première partie, je tâcherai de saisir le projet subversif du droit de la consommation en spécifiant son discours. Dans une deuxième partie, j'essaierai de montrer ce que l'écriture juridique fait à ce discours en l'inscrivant dans le corpus du droit.

\section{Discours}

Le discours du droit de la consommation peut être appréhendé de deux manières. Soit par l'intermédiaire de l'opération de consommation (objet), soit par l'intermédiaire du consommateur (sujet). La manière la plus adéquate de signifier le projet consumériste me semble être la seconde. L'opération de consommation ne saurait, à elle seul, véhiculer un ensemble normatif distinct, dans son principe, du droit qui lui préexiste, droit civil ou common law. Il aura fallu, auparavant, spécifier cette opération de consommation, en indiquer les ressorts particuliers, établir qu'elle implique un régime économique déterminé. Pour soutenir le droit de la consommation, l'opération de consommation devrait être mise en contexte. Et celle-ci implique le rapport consommateur professionnel. Fondamentalement, le discours du droit de la consommation est un discours sur le sujet de droit.

Il mobilise à sa racine deux représentations du sujet : le consommateur, nouveau venu, et le citoyen, figure centrale, fondatrice même, du sujet de droit moderne. Chacun d'eux est soutenu par une structure théorique liée à l'ordre juridique conçu pour une société : celle du citoyen ou celle du consommateur.

23 Jacques Derrida, La voix et le phénomène, supra note 3, aux p. 34-66.

24 Merci à Patrick Glenn pour l'expression. 


\subsection{Le consommateur et le citoyen}

Le droit de la consommation se rapporte à un personnage juridique ${ }^{25}$ appelé consommateur : un nouveau sujet de droit. Celui-ci annonce un changement d'ordre juridique par une éclipse de l'ancien sujet.

\subsubsection{L'hypothèse du citoyen éclipsé par le consommateur}

Soit un ouvrage didactique - le livre de Geraint Howells et Stephen Weatherhil126 : " Ultimately this book is based on the belief that consumer law is not simply a matter of plugging a few gaps in the market system. Consumer law raises issues that are central to the determination of how our society views the citizen [...] Consumer law, we repeat, is part of shaping a society $»^{27}$. (C'est moi qui souligne)

Nous voyons que ce texte ne se répète pas au mot près. Et pour cause. Dans les 92 pages qui séparent la première affirmation de la seconde, il aura été montré l'emprise du droit de la consommation sur le droit de l'économie de marché. Une emprise telle que le droit des contrats, sensibilisé aux échanges auxquels prend part un consommateur, a été ajusté, questionné dans ses principes et sa structure ${ }^{28}$. En outre, le droit de la consommation se déploie dans le droit de la responsabilité pour protéger davantage le consommateur ${ }^{29}$. Il implique de modifier le coût de certains dispositifs juridiques ${ }^{30}$, il pose la question de l'effectivité du droit en interrogeant les recours et l'accès des

25 Mikhaïl Xifaras, « Les figures de la doctrine, Essai d'une phénoménologie des «personnages juridiques dans la doctrine administrative française », dans La doctrine en droit administratif, Travaux de l'Association Française pour la recherche en Droit Administratif - 3, Paris, Litec, 2010, 175, aux p. 175185 : Mikhail Xifaras propose de considérer les auteurs - l'instance narrative - des textes académiques comme des personnages littéraires dont on peut apercevoir des «types». On doit pouvoir traiter un personnage " légalisé » (le consommateur) comme un " personnage juridique » en empruntant à Xifaras son cheminement depuis la théorie littéraire, à des fins cependant un peu différentes. Cette approche littéraire sert ici à indiquer la mise en scène du « sujet de droit ».

26 Supra note 20.

27 A la p. 7 puis à la p. 99

28 Par exemple : «The notion of respect for the parties' bargain as the sole source of legal rights and obligations has declined. In part this flows from a realisation that the notion of contractual freedom, which underpins judicial non-interventionism, is no longer necessarily realistic in the modern consumer transaction where the Relationship between parties is typically economically imbalanced in favour of the supplier. Consumer contract law now embraces rights and obligations inserted into the relationship by judicial activism and by statutory intervention » (p. 18) «this throws up a central question in the modern law of contract: is it realistic or desirable to attempt to maintain a common set of contractual principles applicable to all bargains [...]? » (p. 20) « Legislative intervention is not only concerned to eliminate (some) unfair terms from consumer contracts, but also to add terms into contracts independently of negotiation between the parties» (p. 29), etc.

29 «For the consumer, tort law offers some legal protection beyond the contractual network », ibid. p. 36.

30 Ibid. aux p. 39-46. 
consommateurs à la justice ${ }^{31}$, il entraîne l'Etat et les pouvoirs publics dans la régulation du marché32, il discute le principe de liberté ${ }^{33}$, etc.

L'amplitude du droit de la consommation ayant été indiquée au lecteur, le texte livre une seconde affirmation plus incisive que la première. Le droit de la consommation ne pose plus simplement des questions cruciales pour déterminer la manière dont notre société considère le citoyen. Il contribue à donner sa forme à la société. Il n'est plus seulement envisagé comme un ensemble dont l'instruction fournirait des outils de compréhension du monde social ; il modifie directement celui-ci.

Remarquons alors, à ce moment, la disparition du « citoyen ». Nous sommes en un lieu important du texte : dans la dernière phrase du premier chapitre. C'est une conclusion et une annonce. Le texte clôt un premier propos en restituant l'enseignement de celui-ci. Il augure aussi de ce qui va suivre : des développements consacrés au contenu du « droit de la protection du consommateur » dont on comprend qu'ils ne se désolidariseront pas de ce qui vient d'être dit. Revenons à la comparaison des deux affirmations.

Dans la première, le « citoyen » constituait le point de rencontre du droit de la consommation et de la société, dans la mesure où le premier donnait en partie à voir comment la seconde considérait le citoyen. Sa disparition est lourde de sens dans une affirmation qui se présente comme une « répétition ». En l'absence du « citoyen », la répétition ne peut concerner que le rapport entre le droit de la consommation et la société. Mais au passage, ce rapport s'est modifié dans le sens d'une action du droit de la consommation sur la société.

Et si le citoyen n'était pas, au départ, le point de rencontre du droit de la consommation et de la société mais un obstacle à leur relation ? L'effacement du citoyen laisserait la société se redessiner sous l'influence du droit de la consommation. Le citoyen doit disparaître au moment où le droit de la consommation s'établit. Quels sont les enjeux de cette disparition?

\subsubsection{Enjeux}

Sous la catégorie " citoyen » s'avance la figure totalisante du sujet de droit : 1'individu constitué par le droit et constituant le droit, tout à la fois. En effet, la catégorie « citoyen » est faite de droit. La communauté politique à laquelle le citoyen appartient le marque de son droit propre et lui donne son nom (la cité est civitas : l'ensemble des citoyens $)^{34}$. Le citoyen voit son humanité doublée par l'appartenance à la cité qui le

31 Ibid. aux p. 46-49.

32 Ibid. aux p 49-76.

33 Ibid. aux p 49-76.

34 Gaius : «Tous les peuples policés [Omnes populi, qui legibus et moribus reguntur] se gouvernent en partie par le droit commun à tous les hommes, et en partie par un droit qui leur est propre ; car quand une nation [populus] se fait un droit, il lui devient particulier, et on l'appelle droit civil », Digeste, I, I, 9 (livre I des Institues de Gaius), trad. Henri Hulot et al., Les cinquante livres du Digeste ou des Pandectes de l'Empereur Justinien, Metz, 1803. 
constitue comme tel ${ }^{35}$. Comme dit Jhering, le citoyen dispose du «droit de cité $»^{36}$. En outre, c'est bien le citoyen qui constitue la cité - la civitas qui est aussi l'Etat-, et avec elle, le droit. Le geste de soumission à l'autorité de l'Etat, par lequel le citoyen s'établit comme sujet ${ }^{37}$, est lui-même constitutif de 1'Etat, c'est-à-dire d'une forme du droit : «Un Etat (civitas) est la réunion d'une multiplicité d'hommes sous des lois juridiques [...] Les membres d'une telle société (societas civilis), c'est-à-dire d'un Etat, réunis pour légiférer, s'appellent citoyens (cives) ${ }^{38}$. Ajoutons que le sujet est capable de produire le droit en usant de sa raison.

Derrière le citoyen, il y a une société et son droit. La présence du citoyen dans le texte ouvre celui-ci sur l'ordre social gardé par le droit. De quel droit s'agit-il ? Le droit propre de la société civile, le droit indiscutable au moment où l'on parle. J'en viens alors à l'essentiel.

L'effacement du citoyen au moment où s'affirme l'effet du droit de la consommation sur la société entraine l'effacement de l'ordre civil (au sens de l'ordre de la citoyenneté) qui constituait préalablement la société dont il est question. Un tel effacement libère l'espace textuel du sujet de droit. Il implique donc l'émergence d'un sujet de droit alternatif au citoyen, un autre sujet constitué-constituant du droit. Le consommateur pourrait l'incarner. Le texte ne l'affirme pas, marquant une hésitation, ou jugeant qu'il a suffisamment exposé le droit dont il traite : Consumer Protection Law. La langue anglaise pourrait d'ailleurs apparaître comme le véhicule inattendu de la mise en question du citoyen. Elle ne parle pas, en effet, de « droit de la consommation » mais bien mais de « droit du consommateur ». Le sujet du droit se trouve inévitablement engagé dans le texte.

\subsection{Les structures théoriques $d u$ consommateur et $d u$ citoyen}

Le droit de la consommation menace 1'ordre juridique dominant, non pas en tant qu'ensemble normatif spécifique ${ }^{39}$ mais, plus radicalement, en bousculant la figure du sujet de droit. Tout a commencé par la représentation d'un nouvel être social qui pourrait prétendre à une prise de possession du sujet de droit : le consommateur.

35 Ulpien : « Le droit civil est celui qui ne s'écarte pas totalement du droit naturel et du droit des gens [défini par Ulpien comme le droit des hommes : « le droit des gens est celui dont se servent les hommes : il diffère du droit naturel en ce que celui-ci et propre à tous les animaux ; celui-là n'a lieu qu'entre les hommes ", Digeste, I, I, 1,4], sans leur être cependant asservi. Ainsi, ajouter ou retrancher quelque chose au droit commun, c'est établir un droit particulier à un peuple, qu'on appelle droit civil », Digeste, I, I, 1, 6 .

36 L'évolution du droit (Zweck im Recht), trad. Olivier de Meulenaere, Paris, Maresq Aîné, 1901, à la p. 45.

37 Thomas Hobbes, Du citoyen, Présentation et traduction par Philippe Crignon, Paris, GF Flammarion, 2010, p. 163-164.

38 Emmanuel Kant, Métaphysique des mours II, Doctrine du droit, Doctrine de la vertu, traduction, présentation, bibliographie et chronologie par Alain Renault, Paris, GF Flammarion, 1994, aux p. 128-129.

39 Rappr. Thierry Bourgoignie, «Un droit de la consommation est-il encore nécessaire en 2006 ? » dans Thierry Bourgoignie, dir., Regards croisés sur les enjeux contemporains du droit de la consommation, Cowansville, Editions Yvon Blais, 2006, 1 : « Le débat sur l'utilité du droit de la consommation en 2006 ne doit pas se limiter à un débat de type instrumental qui s'interrogerait sur la localisation des règles de droit de la consommation au sein de l'arsenal juridique $[\ldots] »$. 
Nous parlons d'une construction intellectuelle dont la structure théorique apparait étonnamment semblable à celle du citoyen comme sujet de droit. Mais une telle construction se dresse contre celle du citoyen. Le « consommateur» donne corps à une critique inaugurale du sujet de droit. Considérons d'abord la construction des catégories « citoyen » et « consommateur».

\subsubsection{La construction des catégories}

Le « citoyen » et le « consommateur » se construisent à partir du sujet. Je ne crois pas nécessaire d'insister sur l'importance du rapport entre le sujet et le citoyen, non plus que sur l'ampleur du questionnement portant sur ce rapport, dans la philosophie ${ }^{40}$. Restonsen à l'idée qu'une catégorie du citoyen comme sujet de droit est liée à la « constitution philosophique du sujet moderne $»^{41}$.

Quant aux consommateurs, ils sont entendus « avant tout comme des individus, c'est-à-dire des personnes au sens plein et noble du terme $»^{42} ;$ 《 le consommateur n'est pas seulement cet incapable qu'évoquait Portalis dans son discours, c'est un homme qui est titulaire de droits qui lui sont reconnus parce qu'il est consommateur $»^{43}$. De telles affirmations indiquent un rapport essentiel, pour la constitution du droit de la consommation, entre l'individu et son droit. Le droit de la consommation procède du consommateur et non l'inverse. De la même manière, le droit moderne prenait sa source dans l'individu homme, sujet, citoyen ${ }^{44}$.

Considérons deux séries de textes introductifs. L'une, dans la colonne de gauche, prépare l'exposé des principes politiques ou juridiques garants du " gouvernement dans la liberté $»^{45}$, l'autre, dans la colonne de droite, prépare l'étude du droit de la

40 Pour s'en faire une idée, Etienne Balibar, Citoyen sujet et autres essais d'anthropologie philosophique, Paris, P.U.F., 2011.

41 Etienne Balibar, ibid. à la p. 5 ; voir aussi l' « ouverture », aux p. 35-87.

42 Guido Alpa, "La sécurité du consommateur - Rapport général », dans Le consommateur, t. LVII des Travaux de l'Association Henri Capitant, Journées colombiennes, 2007, Bruylant, LB2V, à la p. 277.

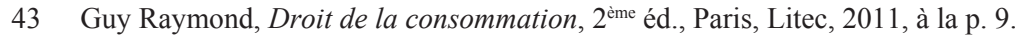

44 J'ai conscience de simplifier. Voir Etienne Balibar, supra note 40 aux p. 43-44: « si le citoyen vient après le sujet, c'est au titre d'une réhabilitation, voire d'une restauration (impliquée dans l'idée même de révolution) : c'est que le sujet n'est pas l'homme originaire et que, contrairement à la thèse de Bossuet, les hommes « naissent », non pas « sujets » mais « libres et égaux en droits. Dans la réponse de fait dont nous disposons déjà $[\ldots]$ est aussi contenue toute la difficulté d'une interprétation qui fait du « sujet » un donné non originaire, un commencement qui n'est pas (et ne peut pas être) l'origine. Puisque l'origine ce n'est pas le sujet mais l'homme [...] la question du sujet, autour de laquelle pivote la révolution copernicienne, est immédiatement caractérisée comme question de droit (quant à la connaissance, quant à l'action). Dans cette question de droit vacille la représentation de l' "homme » dont on disait plus haut qu'il forme l'horizon téléologique du sujet : ce qu'il s'agit de trouver sous son nom n'est pas l'homme de fait [...] mais l'homme de droit [...] dont l'autonomie correspond à la position d'un « législateur universel ». Ce qui, coupant au plus court, nous ramène à la réponse évoquée ci-dessus : après le sujet (subjectus) vient le citoyen. Mais ce citoyen est-il immédiatement ce que Kant nommera sujet (Subjekt) ? Ou bien ce dernier n'est-il pas plutôt la réinscription du citoyen dans un espace philosophique et, par-delà, anthropologique, qui évoque encore en le déplaçant le défunt sujet du prince ?».

45 Voir Lucien Jaume, Les origines philosophiques du libéralisme, Paris, Flammarion, 2010. 
consommation. Le mouvement qui les anime est identique : il s'amorce depuis l'individu jusqu'à l'objet dont il est question : droit de la guerre et de la paix, droit civil, société civile, droit de la consommation. Il faudra donc s'arrêter sur l'individu fondateur de l'objet; cet individu pris dans un certain rapport avec le monde.

On relèvera la correspondance des champs lexicaux de la société, de l'individu et de la justice. De même, on notera l'identité des motifs:

« la condition des hommes hors de la société civile n'est pas autre chose qu'une guerre de chacun contre chacun $[. .$.$] les hommes veulent tous$ $\underline{\text { sortir de cet état misérable et odieux }}$ [...]

il est advenu que ma patrie, quelques années avant que la guerre civile n'éclate, s'est mise en effervescence autour de questions relatives au droit de souveraineté ou à l'obéissance due par les citoyens [...] j'espérais qu'ayant lu et examiné la doctrine que je présente, vous préfèrerez supporter avec résignation quelques inconvénients dans les affaires domestiques [...] que, mesurant la justice de ce que vous comptez faire non pas à l'aune du discours ou du conseil de particuliers mais aux lois de l'Etat, vous ne tolérerez plus que des ambitieux utilisent votre sang au profit de leur puissance personnelle $»^{46}$.
« La notion de consommateur a toutefois un sens particulier qui s'est élaboré avec la nouvelle société de consommation de l'après-guerre. Elle faisait écho à un constat d'impuissance du consommateur individuel et isolé, d'agir sur le marché pour en faire modifier le comportement en raison de sa faiblesse face aux entreprises puissantes et à une organisation du marché des plus raffinées [...] La pression qui s'exerce sur le consommateur ne connaît pas de répit et celui-ci n'a aucun de pouvoir de s'en évader [...] Suite aux recommandations $\mathrm{du}$ Conseil économique, le gouvernement canadien créait en 1967 le Ministère de la consommation à qui il proposait comme objectif de promouvoir un traitement juste et équitable pour tous les citoyens et de favoriser l'intérêt des consommateurs $»^{47}$.

« La société de consommation, qui apparaît après la Seconde Guerre mondiale, se caractérise par une production en série et une distribution massive de toute une variété de biens d'usage courant. Dans le but d'accroître la production par une demande toujours renouvelée la publicité s'est révélée indispensable pour convaincre le consommateur du

46 Thomas Hobbes, supra à la note 36 à la p. 87 et aux pp 89-90.

47 Nicole L'Heureux, L'identification du consommateur, son besoin de protection, (1979) La revue du barreau du Québec 41 aux pp 44-45 pour la citation. 
Je voyais dans l'univers chrétien une débauche de guerre qui eût fait honte même aux nations barbares [...] on n'observait plus aucun respect, ni du droit divin, ni du droit humain [...]

"Quant à moi, convaincu par les considérations que je viens d'exposer, de l'existence d'un droit commun à tous les peuples, et servant soit pour la guerre, soit dans la guerre, j'ai eu de nombreuses raisons pour me déterminer à écrire sur ce sujet $[\ldots]$

j'ai voulu aussi en même temps me rendre utile par l'étude à laquelle je me suis appliqué, dans ma privée, à cette jurisprudence qu'auparavant j'ai pratiquée [...] que si ceux qui se sont consacrés au culte de la vraie justice entreprenaient de traiter séparément des parties de cette naturelle et perpétuelle jurisprudence [...] on pourrait faire de toutes ces parties réunies un corps complet $»^{48}$.

«Pour éviter cet état de guerre [...] les hommes ont formé des sociétés et ont quitté l'état de nature : car s'il y a une autorité, un pouvoir sur la terre, auquel on peut appeler, l'état de guerre ne continue plus, il est exclu, et les différends doivent être décidés par ceux qui ont été revêtus de ce pouvoir caractère nécessaire des nouveaux biens. Par divers moyens elle force la vente et tente d'emporter l'assentiment $\mathrm{du}$ consommateur [...] L'incitation au crédit devient également de plus en plus présente et favorise, dans plusieurs cas, le surendettement de certains consommateurs [...] la gamme de plus en plus étendue et complexe des produits qui inondent le marché est une source de dangers ou de risques pour la santé ou la sécurité du consommateur [...] ces transformations économiques et sociales se traduisent sur le plan juridique par de nouveaux rapports caractérisés par un déséquilibre entre les partenaires économiques [...] inégalité du pouvoir de négociation [...] disparité des connaissances [...] déséquilibre dans les ressources [...] Au milieu des années 1960, le mouvement consumériste a amené les gouvernements à mettre à mettre en place d'importantes législation [...] le consommateur a droit à une opération juste et honnête ${ }^{49}$.

« [...] de façon générale, les consommateurs sont en situation de faiblesse $[\ldots]$ la situation est particulièrement grave pour les consommateurs appartenant aux catégories les plus défavorisées, en raison de leur pauvreté, de leur ignorance ou de leur âge. 
[...] Il paraît évident, par tout ce qu'on vient de lire, que la monarchie absolue [...] est, à vrai dire, incompatible avec la société civile et ne peut nullement être réputée une forme de gouvernement civil. Car la fin de la société civile étant de remédier aux inconvénients qui se trouvent dans l'état de nature, et qui naissent de la liberté ou chacun est, d'être juge dans sa propre cause : et dans cette vue, d'établir une autorité publique et approuvée à laquelle chaque membre de la société puisse appeler et avoir recours $[\ldots]$ et être obligés d'obéir $\aleph^{50}$.

« L'homme est né libre, et partout il est dans les fers. Tel se croit le maître des autres, qui ne laisse pas d'être plus esclave qu'eux. Comment ce changement s'est-il fait ? Je l'ignore. Qu'est-ce qui peut le rendre légitime? Je crois pouvoir répondre à cette question $»^{51}$
Ainsi apparaît la nécessité de protéger les consommateurs non seulement contre les malhonnêtetés qui sont connues depuis longtemps et qui ne sont heureusement pas fréquentes, mais encore contre les abus de puissance économique, qui sont plus dangereux parce qu'ils sont inhérents au système dans lequel nous vivons et qu'ils ne sont pas toujours perçus par l'opinion publique ${ }^{52}$.

« Mais, encore faudrait-il pour restaurer la primauté du social au sein même de l'économie de marché et non à sa marge, que l'on ose retrouver une véritable éthique des affaires et que l'on hésite pas à affirmer l'existence d'un droit à la consommation [...] Moraliser les pratiques commerciales, faire en sorte que le plus faible ne soit pas écrasé par le plus fort, ce n'est pas assimiler droit de la consommation et droit des incapacités, c'est tout simplement tenter de mettre en place un monde plus juste $\gg{ }^{53}$.

\subsubsection{L'ordre juridique}

Colonne de gauche : des textes caractéristiques de la pensée des Lumières ; le moment de la fondation moderne du gouvernement politico-juridique. En préambule, il faut se souvenir qu'un lien épistémique unit l'individu et le monde. En effet, dans la pensée moderne, le monde s'appréhende par l'intermédiaire de l'individu ; c'est-à-dire le « su-

50 John Locke, Traité du gouvernement civil, traduit de l'anglais, édition revue et corrigée exactement sur la dernière de Londres, Paris, Vollant, 1802 à la p. 61 et à la p. 153.

51 Jean-Jacques Rousseau, Du contrat social, présentation, notes, chronologie et bibliographie par Bruno Bernardi, Paris, GF Flammarion, 2001, p. 46.

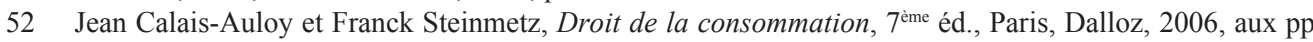
$1-3$.

53 Guy Raymond, supra note 42, à la p. 2. 
jet». La possibilité de connaître est fournie par une réflexion - une méditation ${ }^{54}$ - menée à partir du l'individu. Ainsi, le sujet est source de connaissances. Ceci explique que le droit (droit de la nature et droit des gens) et les procédures sociales de création du droit (production du droit positif) s'attachent, en fin de compte, logiquement à l'individu.

Revenant aux textes, remarquons qu'il est d'abord question d'un monde hypothétique : 1'« état de nature » (Locke), « la condition des hommes hors de la société civile » (Hobbes qui l'appelle par ailleurs « état de nature »). L'état de nature n'a pas à être un fait historique pour remplir sa fonction dans le discours. Il constitue un lieu théorique qui permet de penser l'homme à l'abri du monde social et de découvrir des lois naturelles (de la nature de l'homme). En tant que naturelles, ces lois constituent des vérités scientifiques. Ainsi la liberté naturelle est posée comme vérité ; la loi qui s'en dégage est considérée comme vraie (on pourrait dire « positive »). On peut alors avoir à l'esprit un état juridique initial - il est permis de l'appeler « justice »-dans lequel l'homme dispose de droits naturels, autrement appelés « droits de l'homme $»^{55}$. Ce faisant, une rupture est déjà suggérée entre cet individu, l'homme, et l'état dans lequel il se trouve («l'homme est né libre et partout il est dans les fers »).

Colonne de droite : des extraits de livres de droit de la consommation. On y observe une structure théorique semblable à celle dont on vient de rendre compte. Il s'agit de textes qui précèdent l'analyse technique. Ils visent le propos général du droit de la consommation, cherchent à en justifier l'existence, à en rationaliser le principe. Or si le " déséquilibre » ou l' « inégalité » (L'Heureux et Lacoursière), la " situation de faiblesse » (Calais-Auloy) caractérisent les opérations auxquelles participe le consommateur, c'est qu'une opération équilibrée ou égalitaire au départ est concevable, qu'un renforcement de la position du consommateur dans les rapports auquel il est partie est prescriptible. De même, la possibilité de « restaurer »- de rétablir donc dans son état « la primauté du social » et d'œuvrer à un monde «plus juste » (Raymond) suggère une idée préalable de justice comme finalité du droit ${ }^{56}$. Il y a un état idéal de l'opération de consommation comme il y avait un état de droit naturel. Dans les deux cas, on y puise l'idée de justice.

Après l'individu de l'état de nature vient l'individu en société. Les textes évoquent un sujet lié à un ordre social. Le citoyen assujetti au droit est déjà présent (Grotius signale qu'il livre un traité de droit; de jurisprudence plus précisément). L'entreprise moderne consiste alors à travailler au changement de la condition sociale du citoyen afin qu'advienne un état respectueux des libertés (de l'homme). Le discours de la modernité s'emploie à dénouer le lien qu'il vient de spécifier entre l'individu et le monde. Il faut libérer le citoyen. Amener celui-ci à rompre avec un environnement qui le maintient hors

54 On songe bien sûr à Descartes, Méditations métaphysiques, traduction M. Beyssade, citée par Etienne Balibar, supra note 40 à la p. 91 .

55 Voir Michel Villey, Les origines de la notion de droit subjectif, dans Leçons d'histoire de la philosophie du droit, préface René Sève, Paris, Dalloz, 2002, p. 221-250, spéc. p. 238-250.

56 Le texte fait penser à Gurvitch (L'idée du droit social. Notion et systèmes du droit social. Histoire doctrinale depuis le 17’ème siècle jusqu'à la fin du $19^{\text {ème }}$ siècle, Scientia Verlag, 1972), rappr. Jean-Guy Belley, «Comment l'équité advient-elle dans la société de consommation » dans Pierre-Claude Lafond et Benoît Moore, dir., L'équité au service du consommateur, Cowansville, Editions Yvon Blais, 2010, p. 171. 
de son humanité afin de rejoindre un monde construit à partir de celle-ci. Le contenu des argumentations peut diverger, mais le résultat demeure. Pour Locke, la soumission à un pouvoir absolu ne constitue pas une société civile. Pour Grotius et pour Hobbes, l'individu est livré à un monde social incapable de garantir sa sécurité. A ce moment, il s'agit de résoudre une tension entre un état idéal du droit conçu depuis le sujet (l'homme juridique), et un état autoritaire, liberticide, injuste du droit qui opprime le sujet (le citoyen). La solution offerte est l'état de droit libéral, constitué à partir du sujet de droit moderne, à la fois homme et citoyen.

De la même manière, le droit de la consommation fait suite à la nécessité de protéger l'individu contre les dangers du monde dans lequel il évolue (des dangers « inhérents au système »). Comme la situation du citoyen, celle du consommateur est historicisée ( « la notion de consommateur a toutefois un sens particulier qui s'est élaboré avec la nouvelle société de consommation de l'après-guerre »). Le développement du droit de la consommation trouve une cause dans les sociétés telles qu'elles sont : des sociétés de consommation " apparues » après la Seconde Guerre mondiale. Le consommateur est déjà présent ; il constitue un donné pour la pensée juridique consumériste - comme c'était le cas du citoyen - et il est exposé à une forme d'injustice comparable à celle qui touchait le citoyen : une souveraineté illégitime et une violence. Du sein du marché se développe un pouvoir qui n'est pas fondé sur le droit et assujettit pourtant le consommateur. La violence, ou le risque de violence, naissent de l'incapacité du consommateur à se soustraire aux forces du marché qui le prennent pour cible ; forces auxquelles il ne saurait par ailleurs s'opposer sous peine d'exclusion du monde. Le droit de la consommation se détermine donc sur un impératif libéral : fournir à l'individu les droits qui garantissent sa liberté.

La pensée juridique moderne et la pensée juridique consumériste épousent un même mouvement. Elles prennent position sur le monde, situent l'état de l'individu dans ce monde et rationalisent les principes qui doivent le faire échapper à cet état.

Soulignons que le citoyen et le consommateur sont construits au cours d'une opération typique de la modernité : une critique ${ }^{57}$. Celle-ci distingue, récuse, élabore. Le citoyen est cet individu à qui la critique d'un ordre ancien promet d'échapper ${ }^{58}$ par la reconstitution de son droit propre. Pareillement, c'est la critique d'un monde injuste qui fait émerger le consommateur et son droit.

\subsection{Le droit du consommateur}

Nous disposons maintenant d'une idée de la subversion qu'annonce le droit du consommateur. Le principe de construction de la catégorie « consommateur » - sujet de droit est une critique du droit préexistant. L'ordre juridique dans lequel le droit de la consommation fait irruption, constitue l'état de droit initial, incapable de protéger efficacement l'individu. Le droit de la consommation est autonome dans sa constitution. Il diverge du droit qui n'a pas été pensé à partir du consommateur. Il tend à élaborer

57 Voir Martin Heidegger, Qu'est-ce qu'une chose, trad. Jean Reboul et Jacques Taminiaux, Paris, Gallimard, 1971, p. 129-131.

58 Lucien Jaume, supra note 45, aux p. 13-14. 
un nouveau système de droits en rupture avec l'ordre préalable. Soudainement, le consommateur a des droits et des droits importants ${ }^{59}$. La raison tient à ce qu'en économie de marché, le consommateur forme un point central vers lequel toutes les forces sociales sont ramenées en permanence. Paradoxalement, c'est l'importance du consommateur dans le monde qui explique la précarité de sa situation. Les consommateurs « sont à la fois les rois et les esclaves de cette «société de consommation » qui caractérise les pays développés $\gg{ }^{60}$ Le droit de la consommation se présente comme système de compensation des déséquilibres de la société civile. Le préjudice souffert par le personnage " consommateur » appelle réparation. Le droit de la consommation doit placer le consommateur dans la situation qui devrait être la sienne dans la société de consommation.

La simple idée d'un passage d'une certaine société - appelons-là « civile » - à « la société de consommation » fournit au discours du droit de la consommation l'essentiel de sa charge subversive. Dans la société de consommation, le consommateur éclipse le citoyen. Ce dernier n'y a plus vraiment sa place ; d'autres références que les siennes se sont constituées. La recomposition de la cité sur le point d'advenir rend presque futile la figure du citoyen:

« Le bonheur [...] c'est la référence absolue de la société de consommation : c'est proprement l'équivalent du salut. Mais quel est ce bonheur qui hante la civilisation moderne avec une telle force idéologique ? Il faut réviser là aussi toute vision spontanée. La force idéologique de la notion de bonheur ne lui vient justement pas d'une propension naturelle de chaque individu à le réaliser pour lui-même. Il vient, socio-historiquement, du fait que le mythe du bonheur est celui qui recueille et incarne dans les sociétés modernes le mythe de l'Egalité. Tout la virulence politique et sociologique dont ce mythe est chargé depuis la révolution industrielle et les Révolutions du XIXè siècle s'est transférée sur le bonheur [...] Le principe démocratique est transféré alors d'une égalité réelle, des capacités, des responsabilités, des chances sociales, du bonheur (au sens plein du terme) à une égalité devant l'Objet et autres signes évidents de la réussite sociale et du bonheur. C'est la démocratie du standing, la démocratie de la T.V. de la voiture et de la chaine stéréo, démocratie apparemment concrète, mais tout aussi formelle, qui répond, par delà les contradictions et inégalités sociales, à la démocratie formelle inscrite dans la Constitution [...] Tout le jeu politique du Welfare State et de la société de consommation consiste à dépasser leurs contradictions en accroissant le volume des biens, dans la perspective d'une égalisation automatique par la quantité et d'un niveau d'équilibre final qui serait celui du bien-être total pour tous ${ }^{61}$.

59 « Suddenly, the consumer not only had rights, the consumer had great rights », Douglas J. Whaley, Problems and Materials on Consumer Law, Wolters Kluwer, 2011, à la p. xxiii.

60 Jean Calais-Auloy et Franck Steinmetz, supra note 52 ; une telle idée revient sans cesse dans le droit européen de la consommation; à rapprocher, Balibar citant Louis Althusser à propos du paradoxe de la souveraineté : « Ce Dieu est un Roi-Sujet, c'est-à-dire un Roi-Esclave [...] », supra note 40, à la p. 81.

61 Jean Baudrillard, La société de consommation, ses mythes, ses structures, préface J.-P. Mayer, Denoël, 1970, réimpr. Folio, 2010, aux p. 59-61. 
Dans ce texte de Jean Baudrillard, la société de consommation est bel et bien une société. Par un jeu de substitution des valeurs (le « bonheur » comme « référence absolue » et même fondateur d'une doctrine du « salut ») et de conciliation des intérêts (l'accroissement du volume des biens dans la perspective d'une égalité matérielle réaliserait un compromis entre les forces de production et l'Etat), elle a pris possession de la société civile. L'acte de consommer ne consiste pas qu'une opération sur le marché. Elle indique la participation de l'individu au « système des objets ». L'acte de consommer devient un geste démocratique. Nous voilà prévenus en tout cas : on risque de se tromper en pensant que le déploiement de la société de consommation est limité au commerce des biens et des services.

Le consommateur se présente comme le véritable sujet du monde actuel. Dans ces conditions :

1) Il s'agit moins de faire disparaître le citoyen que de lui soustraire une part de sa capacité à déterminer le droit pour l'attribuer au consommateur. Le monopole que le citoyen (catégorie universelle) exerçait sur la structure théorique du droit disparaît. Il ne semble plus possible de penser le droit de la société contemporaine sans penser au consommateur aux côtés du citoyen. Le consommateur est donc un facteur de division, d'éparpillement, de dislocation du droit.

2) Une certaine valeur du droit est menacée. Si le besoin du droit de la consommation vient de l'insuffisante protection de l'individu par le droit commun, c'est que celui-ci précipite le consommateur dans l'injustice. Qu'il concourt au déséquilibre des rapports juridiques auquel le consommateur est partie. Qu'il soutient les transactions qui font supporter au consommateur des risques excessifs. Qu'il favorise les abus de puissance économique. Qu'il prive de recours une partie de la population, etc ${ }^{62}$. Le droit de la consommation ne désigne pas qu'une carence des ordres juridiques préalables, il révèle leur impossible neutralité. Il indique certaines de leurs orientations. Il (re)politise le droit $^{63}$.

Résumons-nous. Il y a un discours du droit de la consommation conçu autour du consommateur, à savoir l'individu vivant dans la société de consommation. La structure de ce discours rappelle celle du discours juridique de la modernité : celui conçu autour du citoyen - sujet de droit.

62 Voir la brillante introduction de Douglas J. Whaley : «Consumer law is not quite respectable. Embarrassingly, it deals not with the great issues of our day but with the petty ones - issues so small as to be mostly ignored by traditional law. Were consumers duped about the terms of the deal? Caveat emptor. Was the contract filled with unfair terms? Laissez faire (doesn't someone who signs a contract have a duty to read?) Did the car fall apart? The interest rate proves to be deceptive? The credit report wrong? De minimis non curat lex. The court should not bother with such trifles. Our system cannot bear litigating the annoyances of day-to-day life. The flood gates would open and horrors wash ashore.

The problem with this attitude is that consumer issues really do matter. More than that, they matter to everyone because everyone is a consumer... », Problems and Materials on Consumer Law, $3^{\text {ème }}$ éd., Aspen Law \& Business, 2002, à la p. xxi.

63 Thierry Bourgoignie, Eléments pour une théorie du droit de la consommation, Bruxelles, Story-Scientia, 1988, à la p. 185. 
Ce discours indique un droit de la consommation subversif du droit qu'on avait déduit du citoyen. C'est un discours inaugural. Il fonctionne comme un protocole, sans cesse reconduit, indicatif de la nécessité du droit de la consommation: la protection du consommateur. Il en suggère ainsi l'intentionnalité - c'est-à-dire ce qui est visé ${ }^{64}-$ et qui ne peut être que son propre environnement juridique. Il apparaît donc que ce discours annonce une critique radicale du droit dominant par le droit de la consommation. Mais un tel discours ne réalise pas encore cette critique. Il faut que celle-ci prenne forme juridique. C'est à ce moment qu'entre en jeu l'écriture du droit.

\section{Ecriture}

Ecrire le droit de la consommation devrait consister à composer un texte avec les matériaux du droit de la consommation. On atteindrait alors son intentionnalité qui passe par une subversion du droit dominant. Or une telle subversion n'a pas lieu. Il me semble que cela ne tient pas à un échec du projet consumériste mais à sa réalisation par l'écriture juridique.

Le programme subversif annoncé par le droit de la consommation prend forme dans des textes qui rappellent à eux le droit dominant. Ils sont nécessairement ambigus, limités, balancés. L'ordre de l'écriture contraint le droit de la consommation à demeurer subalterne.

Pour l'expliquer, j'utiliserai la construction des subalternes dont parle Gayatri Chakravorty Spivak. Dans son essai Les subalternes peuvent-elles parler ${ }^{65}$, elle montre comment le travail des sciences humaines, conscient d'une dette de reconnaissance à l'endroit des « subalternes » constituées comme telles par l'impérialisme colonial, finit par opérer à l'inverse de ce qu'il annonce. Ainsi :

"Enquêter, parmi les femmes de couleur ou les femmes qui subissent une oppression de classe dans le Premier-Monde ou le Tiers-Monde, sur le travail antisexiste ou, mieux encore, y participer est incontestablement à l'ordre du jour. Nous devons également nous réjouir de la recherche d'informations effectuée en anthropologie, en science politique, en histoire et en sociologie dans ces espaces réduits au silence. Et pourtant, la supposition et la construction d'une conscience, ou d'un sujet, sous-tendent un tel travail et vont, à la longue, converger avec la constitution impérialiste du sujet, mêlant la violence épistémique à l'avancement du savoir et de la civilisation. Et la femme subalterne sera toujours aussi muette $»^{66}$.

64 Paul Ricœur, Du texte à l'action, Essais d'herméneutique II, Paris, Editions du Seuil, 1986, à la p. 30 ; voir aussi Danièle Bourcier et Pierre Mackay dir., Lire le droit - Langue, texte, cognition, Paris, L.G.D.J., 1992 ; Danièle Bourcier et Claude Thomasset, dir., L'écriture du droit face aux technologies de l'information, Diderot Editeur Arts et Sciences, 1996.

65 1988, trad. Jérôme Vidal, Editions Amsterdam, 2009.

66 Supra note 65, aux p. 69-70 (c'est moi qui souligne). 
Ce qu'écrit Gayatri Spivak donne à penser qu'à partir d'un certain niveau de complexité, n'importe quel travail impliquant un jeu de connaissances déterminé recourt à des catégories épistémologiques (dans le texte de Gayatri Spivak ces catégories sont celles de la conscience ou du sujet). C'est-à-dire des catégories inévitables en raison du domaine de savoir dans lequel le travail a lieu. De ce fait, ces catégories peuvent référer mécaniquement à des concepts, des valeurs ou des hiérarchies qui se trouvent en opposition avec les directives que le travail en question s'était lui-même donné. De sorte que par le simple effet du travail, un rapport de domination que l'on pensait devoir modifier se trouve reconduit.

C'est de cette façon que l'écriture du droit a son effet propre, s'intercalant entre le discours qui vise un certain effet de droit et la réalisation de ce dernier. Car même si l'on s'en tient au droit positif (à supposer que cela soit possible), on en vient forcément à manipuler des catégories juridiques qui appartiennent aussi au domaine de la connaissance du droit.

Le niveau de complexité auquel ceci a lieu est rapidement atteint : lorsqu'on entre dans la partie technique du droit, qui est aussi le moment où l'on quitte les préfaces, les introductions, les exposés de motifs; tout ce qui a valeur protocolaire.

Ainsi, le discours du droit de la consommation va converger avec le droit dominant dans l'ordre des formes juridiques. Il est conduit par l'écriture à s'associer aux catégories du droit dominant, à épouser certaines de ses distinctions et à confirmer une partie de ses hiérarchies. Je tâcherai d'indiquer comment cette assimilation se déroule en traitant deux points particuliers : les clauses abusives et l'établissement des distinctions et hiérarchies en droit de la consommation.

\subsection{Les clauses abusives}

Soit la « clause abusive ». « Clause » désigne un élément contractuel du contrat ${ }^{67}$ sans quoi il n'y a pas de «clause ». Mais la clause est abusive ou injuste et donc, hors contrat. Dans son intentionnalité, le dispositif est conforme à la critique du droit dominant annoncé par le discours du droit de la consommation.

Il apparaît hautement subversif : une clause valable selon le processus contractuel commun doit disparaître du contrat. Le droit de la consommation bouscule la hiérarchie des principes et ouvre le front d'une opposition entre consentement et justice ${ }^{68}$, entre justice procédurale et substantielle ${ }^{69}$.

67 Le Vocabulaire juridique indique une « disposition particulière d'un acte juridique ayant pour objet soit d'en préciser les éléments ou les modalités, soit de l'assujettir à un régime spécial, parfois même déro-

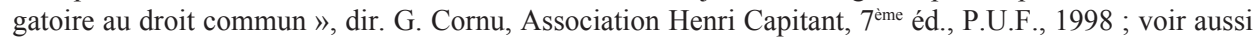
Clause dans le Dictionnaire de droit et de pratique de C.-J. de Ferrière, $3^{\text {ème }}$ éd., revue par M. Boucher d'Argis, Paris, Bauche, 1771.

68 Sébastien Grammond, « La règle sur les clauses abusives sous l'éclairage du droit comparé », (2010) 51 C. de D. 83, spéc. aux p. 88-90.

69 Stephen Smith, Atiyah's Introduction to the Law of Contract, 6th ed., Clarendon Press, Oxford, 2005, p. 296-331; Chris Willett, Fairness in Consumer Contracts - The Case of Unfair Terms, Ashgate, 2007, aux p. 1-73. 
Toutefois, la composition du dispositif dans l'écriture juridique ne dit pas exactement cela. Elle promeut l'ambivalence que crée la juxtaposition de la « clause », c'est-à-dire une convention, et de son caractère abusif qui prescrit la neutralisation de ses effets de droit. L'écriture préserve plutôt qu'elle ne déchire la trame existante du droit.

C'est que l'écriture est programmée la transaction entre l'intentionnalité du droit de la consommation, d'une part, et le droit déjà là, d'autre part. S'agissant de l'intentionnalité, l'objectif de protection du consommateur s'exprime dans la création d'un dispositif assurant l'équilibre contractuel, compensant l'inégalité des capacités de négociation des parties ${ }^{70}$. Il inaugure une technique destinée à suppléer les insuffisances du droit commun qui laisse les parties libres de discuter le contenu de leur accord ${ }^{71}$. Mais il faut aussi traiter ce dispositif en utilisant les mots du droit des contrats. Leur présence inscrit le texte dans la littérature juridique. L'écriture doit, jusqu'à un certain point, faire suite au droit écrit des contrats. Elle doit rappeler un ordre, une linéarité, des concepts, des sources.

L'élimination des clauses abusives concrétise la critique d'un droit autorisant ces clauses. Mais elle se confronte aux prescriptions du code d'écriture du droit des contrats : 1) situer ce que l'on dit par rapport aux concepts fondamentaux : ceux qui se retrouvent le plus souvent dans les textes juridiques 2) respecter la cohérence des textes qui viennent avant celui qu'on est en train d'écrire ; tenir son propre texte dans la linéarité de ce qui précède : écrire droit. Or la lutte contre les clauses abusives implique des dispositifs subversifs de l'ordre du droit des contrats. Ecrire cela exposerait un droit des contrats pris dans la contradiction ${ }^{72}$. Et l'écriture du droit des contrats se réglant sur les textes de droit des contrats, la contradiction serait insurmontable. Le propos juridique risquerait de se désagréger, et son autorité de s'émousser.

Le texte juridique doit résoudre ce problème. Il faut situer les clauses abusives par rapport au cœur du droit des contrats ${ }^{73}$. Limiter l'étrangeté du dispositif. D'où une tendance à traiter l'insertion d'une clause abusive comme un dysfonctionnement de la procédure contractuelle. Une clause abusive est le signe que la conclusion du contrat ne s'est pas déroulée comme elle aurait dû se dérouler. La littérature juridique entretient l'idée selon laquelle le problème réglé par le droit des clauses abusives ne devrait pas se poser si le contrat avait été conclu conformément aux principes fondamentaux du droit des contrats. Le droit des clauses abusives constitue un supplément du droit des contrats écrit avant lui. Il le respecte comme son supérieur.

70 « Inequality of bargaining power was during the 1960's and 1970's a recurring justification for the regulation of unfair terms. This seemed often to reflect the "exploitation thesis" - the thesis that well-organised businesses impose take-or-leave-it terms upon individual consumers, and that consumers are unable to protect themselves against this power », Ian Ramsay, supra note 6, à la p. 156.

71 Nicole L'Heureux et Marc Lacoursière, supra note 51, à la p. 72.

72 On s'autorise à parler de « tension » ou de « crise » (Sébastien Grammond, supra note 68); à évoquer un « conflit» entre les politiques qui sous-tendent le droit des contrats (Jean-Louis Baudouin, Pierre-Gabriel Jobin et Nathalie Vézina, infra note 135, à la p. 130). Mais au terme de l'écriture, le droit des contrats devrait revenir à l'« équilibre» (Baudouin, Jobin et Vézina, à la p. 131 ; Grammond, p. 872).

73 Ce que fait le Code civil du Québec. 
Parcourons rapidement quelques livres de droit civil québécois sur la question. Des obligations, Actes et responsabilité de Maurice Tancelin ${ }^{74}$; Théorie des obligations de Jean Pineau, Serge Gaudet et Danielle Burman ${ }^{75}$; Les obligations de Jean-Louis Baudouin, Pierre-Gabriel Jobin et Nathalie Vezina ${ }^{76}$; Droit des obligations de Didier Lluelles et Benoît Moore ${ }^{77}$.

Tancelin aborde les clauses abusives dans une partie consacrée à l'acte unilatéral. Pineau, Gaudet, et Burman traitent la matière dans un chapitre consacré au contenu du contrat ${ }^{78}$. Baudouin, Jobin et Vezina développent la question dans une partie sur la bonne foi et l'équité (à la suite de l'autonomie de la volonté) ${ }^{79}$. Dans le livre de Lluelles et Moore, un chapitre intitulé « apparence et bonne foi : les clauses abusives » relève d'une partie où il est question de «l'efficacité du contenu contractuel».

Les quatre ouvrages montrent une certaine perplexité quant à la nature du dispositif: l'inconnu ou l'étranger fait irruption en droit civil. « A notre avis, la clause abusive constitue un concept original, possédant des règles et une interprétation qui lui sont propres » ; la reconnaissance de la notion de clause abusive « exige un effort dans la détermination de sa nature juridique ». Il semble aussi délicat de rendre compte du phénomène : que désigne l'expression clause abusive ? La question semble de peu d'importance dans le livre de Tancelin. Celui-ci appréhende les clauses abusives par l'intermédiaire du pouvoir du juge, comme une dérogation au principe de l'intangibilité des contrats. Le problème de la signification de l'expression "clause abusive » est renvoyé aux hypothèses dans lesquelles le juge pourra exercer son pouvoir : contrats de consommation et d'adhésion.

La Théorie des obligations, Les obligations, le Droit des obligations suggèrent le problème et le contournent. Chacun procède par des rapprochements et des distinctions dans le cadre textuel préexistant. Le lecteur est amené à se faire une idée des clauses abusives par la médiation du langage élémentaire du droit des contrats.

Alors, les développements sur les clauses abusives enveloppent le phénomène des clauses abusives. Aborder celui-ci de front supposerait de dire que le droit civil offre des procédures de légalisation des abus ou des injustices en matière contractuelle. Or ceci est bien trop univoque. Les mots du droit doivent conserver une possibilité de produire du droit à venir. Ils doivent demeurer flottants. Insinuer qu'ils indiquent l'injustice (ou la justice) risque de compromettre leur usage. L'écriture dresse un enclos autour des phénomènes avec les mots du droit. Les clauses abusives ne sont pas pensées toutes seules. Il faut les considérer dans l'enclos qui les contient et les spécifie. Les livres s'engagent alors sur la «nature juridique de la notion $\|^{80}$ ou sur la «nature des

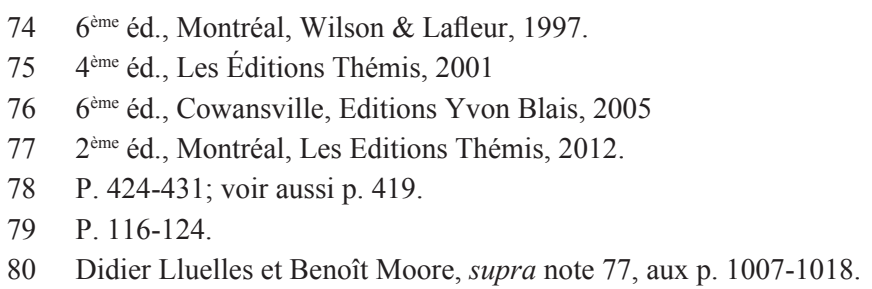


règles sur la clause abusives $»^{81}$. Il s'agit de savoir si une clause abusive s'apparente à la lésion ou à un (autre) vice du consentement, si elle ne constitue pas un cas de révision pour imprévision ou d'abus de droit. Et il s'opère un irrésistible déplacement vers les éléments essentiels du contrat. Un tel déplacement finit par éluder une série de questions : « comment, théoriquement, un contrat valable peut-il comporter une clause abusive ? "; « la sanction des clauses abusives implique-t-elle qu'on puisse obtenir la modification des obligations auxquelles on a pourtant consenti ?»; " peut-on, ou ne peut-on pas, montrer qu'une clause n'est pas abusive en prouvant que le contrat (la clause ?) a été négocié ? ». La question se pose parce que l'idée solitaire de clause abusive éveille le sentiment d'une contradiction. Ce sentiment, inscrit dans les livres de droit des contrats, est cependant repoussé. Il ne conduit pas au constat que l'on pourrait faire : l'incohérence du droit des contrats.

Prenons l'ouvrage de Didier Lluelles et Benoît Moore. L'enchaînement des développements est frappant. La clause abusive commence par être séparée du consentement. L'article 1437 n'a pas pour objet l'altération du consentement contractuel mais « le contrôle du contenu de la clause ». Une première partie de la démonstration s'achève sur « une nette distinction » entre les instruments qui concernent « la réalité du consentement » et l'article 1437 qui se rapporte à « l'équité du contenu ».

Le paragraphe suivant balance cette distinction avec la jurisprudence. Dans certaines affaires, le juge examine l'échange des consentements. Ainsi, lorsqu'il apparait au juge qu'une partie à un contrat d'adhésion a « sciemment et librement » consenti, la clause n'est pas abusive. Les auteurs discutent cette position révélatrice d'une « conception individualiste du contrat ». Ceci expliquerait pourquoi une partie des juges rejette une distinction « aisée sur un plan strictement conceptuel». A ce moment ${ }^{82}$, le texte semble prêt à ouvrir l'interprétation. La lutte contre les clauses abusives devrait être déconnectée de la question de l'échange des consentements. Quelque chose s'annonce : une subversion. Le juge aurait le pouvoir d'annuler une clause valable selon le droit commun. Une clause dont on sait, ou dont il n'est pas contesté, qu'elle a été consentie ; une clause qui se trouve dans le champ du consentement contractuel. Le texte est proche de faire apparaitre un principe de déstabilisation: un contrat valablement formé parce que toutes les conditions requises sont réunies pourrait voir son efficacité compromise en justice. La question est alors de savoir comment justifier l'éradication d'une clause acceptée ? En mobilisant l'idée de clause injuste ${ }^{83}$.

Pourtant, le paragraphe qui clôt cette première partie met fin au mouvement. Il revient juste avant le moment de l'antinomie. Ainsi :

81 Jean-Louis Baudouin, Pierre - Gabriel Jobin et Nathalie Vézina, p. 116-119.

82 Aux p. 1008-1009.

83 Les auteurs citent à cet endroit Adrian Popovici qui insiste: « Même consenties pleinement, les clauses abusives sont visées par l'article 1437. C'est au nom d'un principe supérieur de justice contractuelle que le juge est carrément autorisé à refaire partiellement le contrat qui n'est plus la loi des parties ", Le Code civil et les nouveaux contrats d'adhésion, Conférence Meredith, Lectures, 1992, 139, à la p. 149 pour la citation. 
« S'il est vrai qu'une clause nettement défavorable à l'adhérent fait partie du contrat, c'est bien parce que celui-ci n'y a pas réellement consenti. Certes, la clause abusive concerne l'exécution du contrat, et sa sanction touche la force obligatoire du contrat. Il n'en demeure pas moins que son existence découle d'un manquement à la bonne foi, dans le contexte de la rédaction du contrat - donc de sa formation. Ce raisonnement, rappelant celui que tiennent les partisans de la lésion-vice du consentement, nous ramène de nouveau à la théorie des vices du consentement. »)

Le texte réduit la fracture possible entre le droit des clauses abusives et le droit des contrats. Il faut alors revenir à une idée familière: la présence d'une clause abusive est le signe d'un échange des consentements défectueux. Ce n'est pas suffisant. Il faut encore, conformément à l'ordre de l'écriture du droit des contrats, distinguer la clause abusive des dispositifs déjà susceptibles de subversion.

Dans un système qui n'admet pas la lésion comme cause générale de rescision des conventions, la clause abusive doit être séparée de la lésion ${ }^{84}$. «Faute de quoi, l'article 1405, consacrant le caractère exceptionnel - et ponctuel - du recours à la lésion serait une disposition vide de signification ${ }^{85}$. Cette phrase est remarquable de contention. Elle dit la puissance potentielle de la clause abusive et annonce la nécessité de sa réduction. Le dispositif de lutte contre les clauses abusives pourrait bouleverser le droit des contrats. Si la lésion et les clauses abusives visent le même phénomène, le droit des contrats dirait une chose et son contraire. Les raisons qui maintiennent la lésion à la marge du droit des contrats sont aussi celles qui empêcheront l'idée de clause abusive de se déployer pleinement. Le principe qui interdit de discuter « l'objet même du contrat, l'équilibre économique des prestations, la globalité du contrat » demeure. Un tel principe ne fait luimême que référer à l'idée selon laquelle la commutativité contractuelle est au pouvoir du consentement. De sorte que $1 /$ la rescision n'est pas une cause générale de nullité des conventions ; 2/ 1'admission de la lésion dans certaines hypothèses peut s'expliquer par l'altération supposée du consentement et simplement à la marge par la justice ou d'équité ${ }^{86}$.

La même structure sera utilisée pour distinguer la clause abusive d'un cas de révision pour imprévision, et de l'abus de droit $^{87}$

Le droit des clauses abusives ne peut atteindre qu'indirectement l'essence contractuelle du contrat. De subversif, le dispositif est devenu complémentaire de l'idée de contrat autour de laquelle se regroupe le droit civil : « En fait l'article 1437 appartient à un ensemble de règles d'équité judiciaire par lesquelles le législateur vise à punir les pratiques véritablement choquantes, les stipulations qui s'écartent manifestement des

84 Voir aussi Sylvette Guillemard, «Les clauses abusives et leurs sanctions : la quadrature du cercle », (1999) 59 R. du B. 371.

85 Didier LLuelles et Benoît Moore, supra note 77, à la p. 1012.

86 Voir le cheminement proposé par René Demogue, Traité des obligations en général, I Sources, tome I, aux p. 609-619 ; voir aussi Georges Ripert, La règle morale dans les obligations civiles, 4ème éd., Paris, L.G.D.J., 1949, à la p. 108.

87 Jean-Louis Baudouin, Pierre - Gabriel Jobin et Nathalie Vézina, supra note 81, à la p. 155. 
pratiques contractuelles généralement acceptées par la société, et non pas seulement celles qui sont regrettables $\|^{88}$.

Le droit civil - celui de la société, civitas - était prêt pour les dispositifs de lutte contre les clauses abusives. Tel est l'effet de l'écriture du droit : harmoniser ce qui vient. Et les mots demeurant les mêmes, ce qui vient était, d'une certaine manière, déjà là.

\subsection{Les distinctions et hiérarchies}

Il n'y a pas d'option. Le droit de la consommation s'explique en droit. Ses textes utilisent des mots du droit qui ont déjà eu lieu. Qui ont déjà signifié autre chose ${ }^{89}$. Et lorsque ces textes doivent prendre position contre le droit qui était là avant eux pour respecter leur propre intentionnalité, ils passent par des structures qui reconduisent le droit en question ${ }^{90}$. Par exemple, pour tâcher d'en finir avec la liberté contractuelle du consommateur, il faut référer à la liberté contractuelle ${ }^{91}$. Et il faut alors lui consacrer de l'espace, ouvrir le texte sur elle, la présenter de nouveau. Cette représentation est incontrôlable.

Il est un célèbre exemple : l'invention du « contrat d'adhésion » par Saleilles. En dépit des précautions prises par celui-ci, la formule « contrat d'adhésion » ne peut pas effacer le contrat qu'elle désigne ${ }^{92}$. Le " caractère contractuel » du « contrat d'adhésion » demeure discutable et disponible, contre l'intentionnalité expresse du texte qui l'invente ${ }^{93}$.

Les textes du droit de la consommation réfèrent au droit qui n'est pas le droit de la consommation. Il s'établit, par le simple jeu de la référence, un rapport des droits. Les textes du droit de la consommation participent inévitablement à la constitution des hiérarchies et distinctions du droit dominant. Il s'opère alors un nouveau repli de la

88 Jean-Louis Baudouin, Pierre - Gabriel Jobin et Nathalie Vézina, ibid.

89 Voir Christian Atias, Epistémologie juridique, Dalloz, 2002, p. 84-86.

90 Encore un rapprochement : «Un phonème ou un graphème est nécessairement toujours autre, dans une certaine mesure, chaque fois qu'il se présente dans une opération ou une perception, mais il ne peut fonctionner comme signe ou langage en général que si une identité formelle ne permet de le rééditer et de le reconnaître », Jacques Derrida, supra note 3 à la p. 57.

91 Pierre - Claude Lafond, « Pour en finir avec la liberté contractuelle du consommateur », dans Benoît Moore, dir., Mélanges Jean-Louis Baudouin, Editions Yvon Blais, 2012, p. 483-498.

92 Peut-être Raymond Saleilles avait-il conscience de l'empire incontrôlable du mot contrat, en proposant de désigner certains phénomènes comme « contrats d'adhésion », « faute de mieux »? Il a fait beaucoup en tout cas pour y soustraire son texte : « il n' y a de contrat que là où [...] sans doute il y a contrats et contrats [...] il y a de prétendus contrats qui n'ont du contrat que le nom [...] c'est le cas de tous ces contrats qui revêtent comme un caractère de loi collective et qui, les Romains le disaient déjà, se rapprochent beaucoup plus de la Lex que de l'accord des volontés [...] mais, laissant de côté tout ce qui concerne ces prétendus contrats qui ne sont au fond que des manifestations unilatérales de volontés parallèle [...] », De la déclaration de volonté, Contribution à l'étude de l'acte juridique dans le Code civil allemand, Paris, 1901, aux p. 229-230.

93 Sur ce thème, Claude Masse, « Fondement historique de l'évolution du droit québécois de la consommation », dans Pierre-Claude Lafond, dir., Mélanges Claude Masse, En quête de justice et d'équité, Cowansville, Editons Yvon Blais, 2003, 39, spéc. aux p. 107-118. 
subversion annoncée par le droit de la consommation. On peut distinguer entre plusieurs ordres de distinctions et hiérarchies.

Un ordre topologique. Le droit de la consommation n'échappe pas à un rapport de situation avec l'autre droit. D'entrée de jeu. Sitôt énoncé le « droit de la consommation » - « Consumer Protection Law », on réfère et on spécifie. On réfère au droit qui n'est pas du droit de la consommation et qui se trouve ailleurs. On spécifie un domaine qui est, au moins, un domaine d'écriture. Sous cette double contrainte, le texte de droit de la consommation ne parvient pas à simplement être tel qu'il est. Ou même strictement analytique - traitant les éléments du droit de la consommation comme données et produisant des ensembles d'énoncés autonomes. Au contraire, les textes sont tenus de respecter la référence. Celle-ci agit par rétroaction sur leur contenu. Même un ouvrage voulant se tenir au plus près de la matière empirique ou positive du droit ne peut économiser une allusion au fait qu'il opère dans un domaine spécifique ${ }^{94}$. Or, spécifier implique de distinguer. Etymologiquement, il s'agit de montrer le genre et l'espèce.

Ainsi, le corpus de droit de la consommation se dispose lui-même à côté d'un autre corpus juridique. Les références jouent dans les deux sens: elles rappellent le droit dominant pour en questionner, en limiter, en adapter les effets, mais aussi pour s'y rattacher ${ }^{95}$. Elles l'indiquent comme territoire initial. A son tour, le droit dominant est lié au droit de la consommation. Ainsi, si nous vivons bien dans une société de consommation, son droit n'est pas seulement le droit de la consommation, mais un ensemble plus vaste. Le consommateur n'a pas renversé la figure du sujet de droit. Le citoyen est toujours là ${ }^{96}$.

Une hiérarchie du savoir. Le droit de la consommation vient après. Il faut déjà savoir du droit pour aborder le droit de la consommation. Les connaissances élémentaires dont les facultés de droit instruisent les étudiants ne sont pas empruntées au droit de

94 La préface aux Consumer Protection Law Developments y parvient presque jusqu'à la fin du texte où se trouve l'allusion au domaine du droit en question : «CPLD is the product of an extensive volunteer effort by many capable individuals who have helped shape the law in this area » (c'est moi qui souligne), August T. Horvath et John E. Villafranco, ABA Section of Antitrust Law, Consumer Protection Law

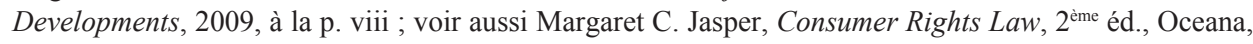
New-York, 2008.

95 «Nous avons vu que le droit contractuel de la consommation s'est construit en marge du code et a été influencé par un grand nombre de sources étrangères. Cela pourrait laisser penser qu'il se situait naturellement à l'extérieur du droit civil, qu'il est important qu'il s'harmonise au droit du marché nord-américain, qu'il en prenne la forme si ce n'est le fond. Ce phénomène n'est pas unique [...] Mais le phénomène est encore plus fort pour le droit de la consommation du fait qu'il demeure à l'extérieur du Code et que la nature des liens entre le droit commun des contrats et le droit de la consommation a toujours fait l'objet d'un vif débat. Pourtant ces liens existent [...] Pourtant malgré ces liens [...] détachement [...] rattachement $[\ldots] »$ B. Moore, « Autonomie ou dépendance : réflexions sur les liens unissant le droit contractuel de la consommation au droit commun », dans Le droit de la consommation sous influences, dir. P.-C Lafond, Editions Yvon Blais, 2007, p. 1-31, p. 11-13 pour la citation.

96 Et le citoyen fait naturellement son retour au texte, formant une limite au droit du consommateur : « The future offers great opportunities for consumers but consumer law must ensure them the freedom to choose how to exploit these possibilities without danger to themselves, their fellow citizens or their environment », Geraint Howells et Stephen Weatherhill, supra note 20, aux p. 660-661. 
la consommation ${ }^{97}$. Le mouvement du savoir juridique part du droit dominant, et se poursuit vers des droits plus composites ${ }^{98}$. L'appréhension du droit de la consommation a lieu dans un cadre épistémologique tracé par le droit dominant.

Suivre le droit de la consommation au plus près de son intentionnalité impliquerait une rupture épistémologique, comme l'a montré Jean-Guy Belley, ou une historicisation radicale, comme l'a montré Claude Masse ${ }^{99}$. Dans les deux cas, c'est l'ordre intime du savoir juridique qui se trouve déstabilisé. Mais de telles opérations auront du mal à préserver leur intégrité sauf à se dégager des contraintes de l'écriture du droit et à supporter leur propre déplacement hors de la littérature juridique. Sinon, il faudra supporter le jeu de la référence. Celui-ci ramène dans le texte des éléments susceptibles de préserver le lecteur de la radicalité de l'historicisation ou de la rupture épistémologique. Dans le texte de Claude Masse, cet élément est le contrat d'adhésion qui ouvre sur le contrat qu'il n'est pas. Le texte de Jean-Guy Belley me semble quant à lui aller très loin sur le trajet de la rupture. Il parvient à soustraire le contrat dont parle la Loi sur la protection du consommateur du champ de connaissance civiliste. Il lui faut cependant garantir son propre statut littéraire, ce qu'il fait à la dernière extrémité. Il propose une juxtaposition du contrat en question et celui du droit civil. Une telle juxtaposition est stratégiquement orientée. Elle ne saurait pourtant dissoudre une catégorie essentielle du savoir juridique. Le contrat civiliste est alors préservé dans un geste quasi taxidermiste, mais qui ne peut éviter de le restaurer comme catégorie élémentaire ${ }^{100}$.

En souscrivant à cette topologie et à cette hiérarchie, l'écriture du droit de la consommation achève de contrebalancer la subversion de ce dernier dans un principe de spécialisation. Le droit de la consommation est un droit spécial ${ }^{101}$. On préfèrera peutêtre dire «sectoriel », ou dire «statutaire ». Cela revient au même : le droit de la

97 On ne trouve guère de référence au droit de la consommation dans les ouvrages d'introduction au droit canadien, voir Richard Yates, Ruth. W. Yates, Penny Bain, Introduction to Law in Canada, $2^{\text {nd }}$ ed., 2000, Prentice Hall Allyn and Bacon Canada p. 247 ; également Gerald L. Gall, Canadian Legal System, Carswell, 1995 ; Stephen M. Waddams, Introduction to the Study of Law, 7ème éd., Carswell 2010.

98 Pour se représenter ce mouvement, voir par ex. l'ouvrage Pierre-Claude Lafond et Benoît Moore, dir., L'équité au service du consommateur, Cowansville, Editions Yvon Blais, 2010.

99 Il me semble important de lire ces deux textes à la suite : Claude Masse, «Fondement historique de l'évolution du droit québécois de la consommation », supra note 93 ; Jean-Guy Belley, La Loi sur la protection du consommateur comme archétype d'une conception socioéconomique du contrat, supra note 15.

100 «Un juriste plaideur ou juge se réfèrera au Code civil du Québec comme à un dictionnaire alphabétique et analogique. Il y trouvera la définition, la signification et la portée du contrat de droit commun en tant que mot du vocabulaire juridique, concept de la pensée juridique et institution ou ensemble de règles permettant d'apprécier la responsabilité juridique d'un contractant en contexte judiciaire... Un commerçant ou un consommateur, quant à lui, se réfèrera à la Loi sur la protection du consommateur comme à un guide technique indiquant à quel écrit standard doit ressembler le contrat de consommation et quel processus doit être suivi par chaque contractant pour obtenir légitimement l'avantage socioéconomique qu'il convoite », J.-G. Belley, supra note 15, à la p. 146.

101 La question a beaucoup intéressé la doctrine française. Parmi une abondante littérature : Yves Picod et Hélène Davo, supra note 17, aux p. 4-5 et les références citées ; Jean Beauchard, « Remarques sur le Code de la consommation », dans Jean Beauchard et Pierre Couvrat, dir., Droit civil, procédure, linguistique juridique. Ecrits en hommage à Gérard Cornu, P.U.F., 1994, p. 9 ; Nathalie Sauphanor, L'influence du droit de la consommation sur le système juridique, thèse, L.G.D.J., 2000, spéc. aux p. 224-226. 
consommation est un Autre que le droit commun. Mais un Autre que le droit commun permet d'appréhender. Et de définir, de contenir, d'achever ${ }^{102}$. Le consommateur est non seulement une partie faible, mais aussi une catégorie juridique faible. Seul, il n'a pas d'avenir. Il annonçait un droit subversif ; l'écriture le réinscrit dans l'harmonie des systèmes au sein desquels il avait fait irruption.

\section{Conclusion}

L'écriture juridique organise l'ambivalence du droit de la consommation. Elle opère une transaction entre le discours consumériste inaugural et le corpus ou codex juridique dominant. Cette transaction a d'abord pour effet de fondre le propos subversif du droit de la consommation dans le texte juridique. Parmi les signes d'une telle opération, il faut souligner ici les plus spectaculaires : 1) la création, le maintien, le renouvellement des partitions du droit : droit commun / droit spécial, common law / statutes, droit civil / droit de la consommation, code civil / code de la consommation... 2) le traitement des questions consuméristes par la médiation des catégories et des concepts fondamentaux du droit (tels que contrat, délit, transfert de propriété, consentement, consideration, bien...) mais aussi de tous les mots et expressions si familiers aux juristes qu'ils semblent à voir avec le cœur de la connaissance du droit (moyen de droit, régime juridique, adjudication, règle, jurisprudence, recevabilité...).

Cette transaction a ensuite pour effet de confier au texte juridique la mémoire de la critique adressée au droit. Celle-ci est enfouie, silencieuse, implicite, mais elle demeure. Le texte contient parfois des indicateurs de la transaction qui a eu lieu. Lorsque le droit s'exprime de manière contradictoire : clause abusive, contrat d'adhésion.

Ainsi, l'écriture juridique installe et maintient le droit de la consommation dans un statut de subalterne créé pour lui. Pareillement au personnage consommateur, qui ne peut finalement exister qu'à l'intérieur de la limite tracée par le personnage citoyen, le droit de la consommation se tient dans l'ombre du droit dominant.

Ce n'est pas inéluctable. En indiquant ici la part que l'on peut attribuer à l'entreprise de formalisation du droit par l'écriture dans cette reconduite permanente du droit de la consommation aux marges du droit dominant, j'espère avoir suggéré des possibilités d'infléchissement de cette tendance. Si on trouve dans l'idée de droit de la consommation des motifs de le faire. L'une de ces possibilités passe certainement par la prise de conscience d'un objet d'étude alternatif pour les juristes : l'écriture juridique ou la textualisation du droit.

102 Très nettement, Laurent Aynès, « Le consommateur », Rapport de synthèse des Journées colombiennes de l'Association Henri Capitant en 2007, Travaux de l'Association Henri Capitant, t. LVII, Le consommateur, Bruylant - LB2V, 2010, p. 11-16, aux p. 15-16 pour la citation. 


\section{Bibliographie}

Atias, Christian, Questions et réponses en droit. Paris, P.U.F., 2009.

Atias, Christian, Epistémologie juridique. Dalloz, 2002.

Balibar, Etienne, Citoyen sujet et autres essais d'anthropologie philosophique. Paris, P.U.F., 2011.

Baudouin, Jean-Louis, Jobin, Pierre-Gabriel, et Vézina, Nathalie, Les obligations. $6^{\mathrm{ème}}$ éd., Cowansville, Editions Yvon Blais, 2005.

Baudrillard, Jean, La société de consommation, ses mythes, ses structures. Préface J.-P. Mayer, Denoël, 1970, réimpr. Folio, 2010.

Beauchard, Jean, " Remarques sur le Code de la consommation », dans Jean Beauchard et Pierre Couvrat, dir., Droit civil, procédure, linguistique juridique. Ecrits en hommage à Gérard Cornu. P.U.F., 1994.

Belley, Jean-Guy, « La Loi sur la protection du consommateur comme archétype d'une conception socioéconomique du contrat ", dans Pierre-Claude Lafond, dir., Mélanges Claude Masse, En quête de justice et d'équité. Cowansville, Editions Yvon Blais, 2003.

Bourcier, Danièle, et Mackay, Pierre, (dir.), Lire le droit - Langue, texte, cognition. Paris, L.G.D.J., 1992.

Bourcier, Danièle, et Mackay, Pierre, (dir.), L'écriture du droit face aux technologies de l'information. Diderot Editeur Arts et Sciences, 1996.

Bourgoignie, Thierry, (dir.), Regards croisés sur les enjeux contemporains du droit. Cowansville, Editions Yvon Blais, 2006.

Bourgoignie, Thierry, Eléments pour une théorie du droit de la consommation. Bruxelles, Story-Scientia, 1988.

Cornu, Gérard, (dir.), Vocabulaire juridique. Association Henri Capitant, $7^{\text {ème }}$ éd., P.U.F., 1998.

Cumyn, Michelle, Les catégories, la classification et la qualification juridiques : réflexions sur la systématicité du droit. (2011) 52 Les Cahiers de Droit 351.

Cumyn, Michelle, " La classification des catégories juridiques en droit comparé. Métaphores taxonomiques ", (2008) R. du N. 1 (erratum) (numéro spécial du Xe Congrès de l'Association internationale de méthodologie juridique sur la sécurité juridique).

Demogue, René, Traité des obligations en général, I Sources, tome I. Paris, Arthur Rousseau, 1923.

Derrida, Jacques, De la grammatologie. Paris, Les Editions de Minuit, 1967.

Derrida, Jacques, La voix et le phénomène, Introduction au problème du signe dans la phénoménologie de Husserl.

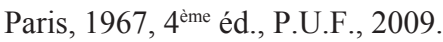

Derrida, Jacques, La dissémination. Seuil, 1993.

Calais-Auloy, Jean, et Steinmetz, Franck, Droit

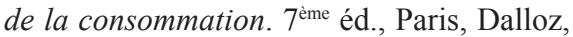
2006.

Ferrière de, C.-J., Dictionnaire de droit et de pratique. $3^{\text {ème }}$ éd., revue par $\mathrm{M}$. Boucher d'Argis, Paris, Bauche, 1771.

Gall, Gerald L., Canadian Legal System. Carswell, 1995.

Glenn, H. Patrick, Legal Traditions of the World. 4th ed., Oxford University Press, 2010.

Goyard-Fabre, Simone, Re-penser la pensée du droit, Les doctrines occidentales modernes au tribunal de la raison interrogative critique. Paris, Vrin, 2007.

Grammond, Sébastien, " La règle sur les clauses abusives sous l'éclairage du droit comparé ». (2010) 51 C. de D. 83.

Grotius, Hugo, Le droit de la guerre et de la paix. Trad. Paul Pradier-Fodéré, Paris, 1867.

Guillemard, Sylvette, « Les clauses abusives et leurs sanctions : la quadrature du cercle ». (1999) 59 R. du B. 371. 
Horvath, August T., et Villafranco, John E., ABA Section of Antitrust Law, Consumer Protection Law Developments. 2009

Heidegger, Martin, Qu'est-ce qu'une chose. Trad. Jean Reboul et Jacques Taminiaux, Paris, Gallimard, 1971.

Hobbes Thomas, Du citoyen. Présentation et traduction par Philippe Crignon, Paris, GF Flammarion, 2010.

Howells, Geraint, et Weatherhill Stephen, Consumer Protection Law. Ashgate 2005.

Jasper, Margaret C., Consumer Rights Law. $2^{\text {ème }}$ éd., Oceana, New-York, 2008.

Jaume, Lucien, Les origines philosophiques $d u$ libéralisme. Paris, Flammarion, 2010.

Jhering, Rudoph von, L'évolution du droit (Zweck im Recht). Trad. Olivier de Meulenaere, Paris, Maresq Aîné, 1901.

Kant, Emmanuel, Le conflit des Facultés en trois sections. Traduction, introduction et notes par J. Gibelin, 5 ${ }^{\text {ème }}$ éd., Paris, Vrin, 1997.

Kant, Emmanuel, Métaphysique des mours II, Doctrine du droit, Doctrine de la vertu. Traduction, présentation, bibliographie et chronologie par Alain Renault, Paris, GF Flammarion, 1994.

Kristeva, Julia, Etrangers à nous-mêmes. Paris, Gallimard, 1988.

Locke, John, Traité du gouvernement civil. Traduit de l'anglais, édition revue et corrigée exactement sur la dernière de Londres, Paris, Vollant, 1802.

Kennedy, Duncan, The Disenchantment of Logically Formal Legal Rationality, or Max Weber's Sociology in the Genealogy of the Contemporary Mode of Western Legal Thought. (2004) 55 Hastings L. J. 1031.

Le consommateur, t. LVII des Travaux de l'Association Henri Capitant, Journées colombiennes, 2007, Bruylant, LB2V, 2011.

L'Heureux, Nicole, Droit de la consommation. $5^{\text {ème }}$ éd., Cowansville, Editions Yvon Blais, 2000.

L'Heureux, Nicole, L'identification $d u$ consommateur, son besoin de protection. (1979) La revue du barreau du Québec 41.
L'Heureux, Nicole, et Lacoursière, Marc, Droit de la consommation. $6^{\text {ème }}$ éd., Cowansvill, Editions Yvon Blais, 2011.

Llewellyn, Karl. N., The Theory of rules. Edited and with an Introduction of Frederick Schauer, The University of Chicago Press, 2011.

Lluelles Didier, Moore, Benoît, Droit des obligations. $2^{2 \mathrm{ème}}$ éd., Montréal, Les Editions Thémis, 2012

Masse, Claude, « Fondement historique de l'évolution du droit québécois de la consommation », dans Pierre-Claude Lafond, dir., Mélanges Claude Masse, En quête de justice et d'équité. Cowansville, Editons Yvon Blais, 2003, p. 39.

Mélanges Jean-Louis Baudouin, dir. Benoît Moore, Editions Yvon Blais, 2012, p. 483.

Menderson, Desmond, Proximity, Levinas, and the Soul of Law. McGill-Queen's University Press, 2006.

Moore, Benoît, « Autonomie ou dépendance : réflexions sur les liens unissant le droit contractuel de la consommation au droit commun », dans Le droit de la consommation sous influences, dir. P.-C Lafond. Editions Yvon Blais, 2007, p. 1.

Picod, Yves et Davo, Hélène, Droit de la consommation. $2^{\mathrm{ème}}$ éd., Paris, Dalloz, 2010.

Pineau, Jean, Gaudet, Serge et Burman, Danielle, Théorie des obligations. $4^{\mathrm{ème}}$ éd., Les Éditions Thémis, 2001.

Popovici, Adrian, " Le Code civil et les nouveaux contrats d'adhésion ». Meredith Lectures, 1992.

Ramsay, Ian, Consumer Law and Policy, Text and Materials on Regulating Consumer Markets. $2^{\text {nd }}$ ed., Oxford and Portland, Hart Publishing, 2007.

Ramsay, Ian, (editor), Consumer Law in the Global Economy, National and International Dimensions. Ashgate, Dartmouth, 1997.

Raymond, Guy, Droit de la consommation. $2^{\text {ème }}$ éd., Paris, Litec, 2011.

Reinach, Adolf, Les fondements a priori $d u$ droit civil. Traduit de l'allemand par Ronan de Calan, Paris, Vrin, 2004. 
Ricœur, Paul, Du texte à l'action, Essais d'herméneutique II. Paris, Editions du Seuil, 1986.

Ripert, Georges, La règle morale dans les obligations civiles. $4^{\mathrm{èm}}$ éd., Paris, L.G.D.J., 1949.

Rousseau, Jean-Jacques $\mathrm{Du}$ contrat social. Présentation, notes, chronologie et bibliographie par Bruno Bernardi, Paris, GF Flammarion, 2001.

Saleilles, Raymond, De la déclaration de volonté, Contribution à l'étude de l'acte juridique dans le Code civil allemand. Paris, Rousseau, 1901.

Sauphanor, Nathalie, L'influence du droit de la consommation sur le système juridique. Thèse, L.G.D.J., 2000.

Smith, Stephen, Atiyah's Introduction to the Law of Contract. 6th ed., Clarendon Press, Oxford, 2005.

Spanogle, John A., et al., Consumer Law, Cases and Materials. 3ème ed., Thomson West, 2007.

Spivak, Gayatri Charkravorty, Les subalternes peuvent-elles parler. Trad. Jérôme Vidal, Editions Amsterdam, 2009.

Tancelin, Maurice, Des obligations, Actes et

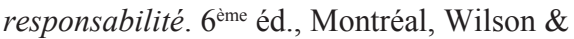
Lafleur, 1997.
Villey, Michel, Leçons d'histoire de la philosophie du droit. Réédition présentée par René Sève, Paris, Dalloz, 2002.

Vukowich, T. William, Consumer Protection in the 21st Century, A global Perspective. Transnational Publishers, 2002.

Waddams, Stephen M., Introduction to the Study of Law. 7ème éd., Carswell, 2010.

Weber, Max, Sociologie du droit. Préface Philippe Raynaud, introduction et traduction par Jacques Grosclaude, Paris, P.U.F., 2007.

Whaley, Douglas J., Problems and Materials on Consumer Law. Wolters Kluwer, 2011.

Willett, Chris, Fairness in Consumer Contracts - The Case of Unfair Terms. Ashgate, 2007.

Xifaras, Mikhaïl, " Les figures de la doctrine, Essai d'une phénoménologie des « personnages juridiques dans la doctrine administrative française ", dans $L a$ doctrine en droit administratif. Travaux de l'Association Française pour la recherche en Droit Administratif -3, Paris, Litec, 2010.

Yates, Richard, Yates, Ruth. W., Bain, Penny, Introduction to Law in Canada. $2^{\text {nd }}$ ed., Prentice Hall Allyn and Bacon Canada, 2000.

\title{
DVIPRASMYBÉS VARTOTOJŲ TEISĖJE: İŽVALGOS DE்L İSTATYMŲ RAŠYMO
}

\section{Vincent Forray}

\author{
McGillio universitetas, Kanada
}

Santrauka. Kiekvienoje teises sistemoje vartotoju teise rodo problemas, kurias ji turetu. spręsti: galios netolygumas, netesingumas, nesąžiningumas. Keliami rimti klausimai dèl teisines sistemos gebejimo kovoti su šiomis problemomis. Tam tikra prasme vartotoju teise atspindi visa teisine sistema.

Analizuojant vartotoju teise kaip teises sistemos dali, dviem aspektais kyla prieštara. Pirmasis aspektas yra tas, kad vartotoju teise formuluojama kaip atskira nuo kitu teisès šaku, kartais kairieji tai teisina per socialinio teisingumo ir rinkos reguliavimo prizmes. Vertinant šiuo diskursu, vartotoju teise gali pasirodyti griannati teises sistemą. Antrasis diskursas api- 
ma vartotoju teise i bendraja privatine teisę. Tokiu aspektu vartotoju teise negriauna teisès sistemos, o integruojasi i ja. Toks skritingas vertinimas gali büti pastebimas tarp pradiniu. vartotoju teises tiklsu ir jos sukurtu rezultatu. Šame darbe nurodoma, jog toks skritingas vertinimas, kuris generuoja vartotoju teises dvilypuma, yra kilęs iš Vakaru teises sistemu tendencijos formalizuoti teise ja itvirtinant teisès aktuose.

Reikšminiai žodžiai: vartotojų teisé, vartotojai, piliečiai, teisinè tvarka, formalizacija.

\title{
AMBIVALENCES IN CONSUMER LAW: REMARKS ON THE EFFECTS OF WRITING THE LAW
}

\author{
Vincent Forray \\ McGill University, Canada
}

Summary. In every legal system, consumer law indicates problems that it is supposed to solve: imbalance, unfairness and unconscionability. It raises important questions about a legal system's pre-existing ability to deal with those problems. In a way, consumer law's very presence in a legal system judges that system.

When considering consumer law as a part of the system, an opposition arises between two kinds of discourse. First, there is a discourse, which constructs consumer law as a legal entity apart from the others, sometimes leftist, founded on a defense of social justice or on market regulation. From this angle, consumer law might easily appear to be subversive. Second, there is a discourse, which results from the effort to accommodate consumer law within the legal tradition's general private law. Under this discourse, rather than disrupting the core private law, consumer law is integrated into it. A gap may thus be noticeable between the initial intentions that sustain consumer law and the effects it produces. The author's claim in this paper is that this gap, which generates ambivalence about consumer law, results from western legal systems' main way of formalizing law in the contemporary era by writing.

Keywords: consumer law, writing, consumer, citizen, critique, legal order, formalization.

Vincent Forray, McGillio universiteto Teisès fakulteto (Monrealis, Kanada) profesorius, Paul-André Crépeau Privatinès ir lyginamosios teisès centro (Monrealis, Kanada) narys. Mokslinių tyrimų kryptys: kritiniai bei transsisteminiai tyrimai sutarčių teisès, deliktų teisès, prievolių teisės bei vartotojų teisès srityse; numanomas politinis, filosofinis bei literatūrinis teisès formų santykinumas ir tai, kas apibūdina teisinị formalizavimą teisininkų raštuose.

Vincent Forray, McGill university, Faculty of Law (Montreal, Canada), Professor; member of the Paul-André Crépeau Center for Private and Comparative Law. Research interests: critical and transsytemic research in contract law, torts law, civil law of obligations and consumer law; studying the scientific, pedagogical and professional jurist's activities; the implicit political, philosophical and literary determination of the legal forms, and what characterizes legal formalization in the jurist's writing. 